\title{
Coupling Index and stocks
}

\author{
Benjamin Jourdain 1 and Mohamed Sbai ${ }^{1}$
}

\begin{abstract}
In this paper, we are interested in continuous time models in which the index level induces some feedback on the dynamics of its composing stocks. More precisely, we propose a model in which the log-returns of each stock may be decomposed into a systemic part proportional to the log-returns of the index plus an idiosyncratic part. We show that, when the number of stocks in the index is large, this model may be approximated by a local volatility model for the index and a stochastic volatility model for each stock with volatility driven by the index. This result is useful in a calibration perspective : it suggests that one should first calibrate the local volatility of the index and then calibrate the dynamics of each stock. We explain how to do so in the limiting simplified model and in the original model.
\end{abstract}

\section{Introduction}

From the early eighties, when trading on stock index was introduced, quantitative finance faced the problem of efficiently pricing and hedging index options along with their underlying components. Many advances have been made for single stock modeling and a variety of solutions to escape from the very restrictive Black \& Scholes model has been deeply investigated (such as local volatility models, models with jumps or stochastic volatility models). However, when the number of underlyings is large, index option pricing, or more generally basket option pricing, remains a challenge unless one simply assumes constantly correlated dynamics for the stocks. The problem then is the impossibility of fitting both the stocks and the index smiles.

We try to address this issue by making the dynamics of the stocks depend on the index. The natural fact that the volatility of the index is related to the volatilities of its underlying components has already been accounted for in the works of Avellaneda et al. 11 and Lee et al. [13. In the first paper, the authors use a large deviation asymptotics valid for small values of the product of the maturity by the square of the volatility to reconstruct the local volatility of the index from the local volatilities of the stocks. They express this dependence in terms of the implied volatilities using the results of Berestecky et al.([4, 3]). In the second paper, the authors reconstruct the Gram-Charlier expansion of the probability density of the index from the stocks using a moments-matching technique. Both papers consider local volatility models for the stocks and a constant correlation matrix but the generalization to stochastic volatility models or to varying correlation coefficients is not straightforward.

Another point of view is to say that the volatility of a composing stock should be related to the index level, or say to the volatility of the index, in some way. This is not astonishing since the index represents

\footnotetext{
${ }^{1}$ Université Paris-Est, CERMICS, Projet MathFi ENPC-INRIA-UMLV. This research benefited from the support of the "Chair Risques Financiers", Fondation du Risque.

Postal address : 6-8 av. Blaise Pascal, Cité Descartes, Champs-sur-Marne, 77455 Marne-la-Vallée Cedex 2.

E-mails : jourdain@cermics.enpc.fr and sbai@cermics.enpc.fr
} 
the move of the market and reflects the view of the investors on the state of the economy. Moreover, it is consistent with equilibrium economic models like CAPM. Following this idea, we propose a new modeling framework in which the volatility of the index and the volatilities of the stocks are related. We show that, when the number of underlying stocks tends to infinity, our model reduces to a local volatility model for the index and to a stochastic volatility model for the stocks where the stochastic volatility depends on the index level. This asymptotics is reasonable since the number of stocks composing an index is usually large. As a consequence, the correlation matrix between the stocks in our model is not constant but stochastic and we show that it is consistent with empirical studies. Finally, we address calibration issues and we show that it is possible, within our framework, to fit both index and stocks smiles. The method we introduce is based on the simulation of SDEs nonlinear in the sense of McKean, and non-parametric estimation of conditional expectations.

This paper is organized as follows. In Section 1, we specify our model for the index and its composing stocks and in Section 2 we study the limiting model when the number of underlying stocks goes to infinity. Section 3 is devoted to calibration issues. Numerical results are presented in Section 4 and the conclusion is given in Section 5 .

Acknowledgements: We thank Lorenzo Bergomi, Julien Guyon and all the equity quantitative research team of Societe Generale CIB for numerous fruitful discussions and for providing us with the market data.

\section{Model Specification}

An index is a collection of stocks that reflects the performance of a whole stock market or a specific sector of a market. It is valued as a weighted sum of the value of its underlying components. More precisely, if $I_{t}^{M}$ stands for the value at time $t$ of an index composed of $M$ underlyings, then

$$
I_{t}^{M}=\sum_{j=1}^{M} w_{j} S_{t}^{j, M}
$$

where $S_{t}^{j, M}$ is the value of the stock $j$ at time $t$ and the weightings $\left(w_{j}\right)_{j=1 \ldots M}$ are given constant:2

Unless otherwise stated, we always work under a risk-neutral probability measure. In order to account for the influence of the index on its underlying components, we specify the following stochastic differential equations for the stocks

$$
\forall j \in\{1, \ldots, M\}, \quad \frac{d S_{t}^{j, M}}{S_{t}^{j, M}}=\left(r-\delta_{j}\right) d t+\beta_{j} \sigma\left(t, I_{t}^{M}\right) d B_{t}+\eta_{j}\left(t, S_{t}^{j, M}\right) d W_{t}^{j}, S_{0}^{j, M}=s_{0}^{j}
$$

where

- $r$ is the short interest rate,

- $s_{0}^{j}$ is the initial value of the stock $j$,

- $\delta_{j} \in[0, \infty[$ is the continuous dividend rate of the stock $j$,

- $\beta_{j}$ is the usual beta coefficient of the stock $j$ that quantifies the sensitivity of the stock returns to the index returns (see the seminal paper of Sharpe [17]). It is defined as $\frac{\operatorname{Cov}\left(r_{j}, r_{I}\right)}{\operatorname{Var}\left(r_{I}\right)}$ where $r_{j}$ (respectively $r_{I}$ ) is the rate of return of the stock $j$ (respectively of the index),

\footnotetext{
${ }^{2}$ The weightings are periodically updated but, as usually assumed, we suppose that, up to maturities of the options considered, they are constant. When updated, they are often chosen proportional to the market capitalizations of the stocks.
} 
- $\left(B_{t}\right)_{t \in[0, T]},\left(W_{t}^{1}\right)_{t \in[0, T]}, \ldots,\left(W_{t}^{M}\right)_{t \in[0, T]}$ are independent Brownian motions,

- the functions $\sigma, \eta_{1}, \ldots, \eta_{M}:[0, T] \times \mathbb{R} \rightarrow \mathbb{R}$ satisfy the usual Lipschitz and growth assumptions that ensure existence and strong uniqueness of the solutions (see for example Theorem 5.2.9 of [12]) :

$$
\begin{aligned}
& \exists K \text { such that } \forall\left(t, s_{1}, s_{2}\right) \in[0, T] \times \mathbb{R}^{M} \times \mathbb{R}^{M}, \\
& \qquad \sum_{j=1}^{M}\left|s_{1}^{j} \sigma\left(t, \sum_{k=1}^{M} w_{k} s_{1}^{k}\right)\right|+\left|s_{1}^{j} \eta_{j}\left(t, s_{1}^{j}\right)\right| \leq K\left(1+\left|s_{1}\right|\right) \\
& \sum_{j=1}^{M}\left|s_{1}^{j} \sigma\left(t, \sum_{k=1}^{M} w_{k} s_{1}^{k}\right)-s_{2}^{j} \sigma\left(t, \sum_{k=1}^{M} w_{k} s_{2}^{k}\right)\right| \leq K\left|s_{1}-s_{2}\right| \\
& \\
& \sum_{j=1}^{M}\left|s_{1}^{j} \eta_{j}\left(t, s_{1}^{j}\right)-s_{2}^{j} \eta_{j}\left(t, s_{2}^{j}\right)\right| \leq K\left|s_{1}-s_{2}\right| .
\end{aligned}
$$

As a consequence, the index satisfies the following stochastic differential equation :

$$
d I_{t}^{M}=r I_{t}^{M} d t-\left(\sum_{j=1}^{M} \delta_{j} w_{j} S_{t}^{j, M}\right) d t+\left(\sum_{j=1}^{M} \beta_{j} w_{j} S_{t}^{j, M}\right) \sigma\left(t, I_{t}^{M}\right) d B_{t}+\sum_{j=1}^{M} w_{j} S_{t}^{j, M} \eta_{j}\left(t, S_{t}^{j, M}\right) d W_{t}^{j}
$$

Before going any further, let us make some preliminary remarks on this framework.

- We have $M$ coupled stochastic differential equations. The dynamics of a given stock depends on all the other stocks composing the index through the volatility term $\sigma\left(t, I_{t}^{M}\right)$. Since there are $M$ linearly independent assets and $M+1$ driving Brownian motions, the market is incomplete.

- Accounting for the dividends is not relevant for all types of indices. Indeed, for many performancebased indices (such as the German DAX index) dividends and other events are rolled into the final value of the index.

- The cross-correlations between stocks are not constant but stochastic :

$$
\rho_{i j}(t)=\frac{\beta_{i} \beta_{j} \sigma^{2}\left(t, I_{t}^{M}\right)}{\sqrt{\beta_{i}^{2} \sigma^{2}\left(t, I_{t}^{M}\right)+\eta_{i}^{2}\left(t, S_{t}^{i, M}\right)} \sqrt{\beta_{j}^{2} \sigma^{2}\left(t, I_{t}^{M}\right)+\eta_{j}^{2}\left(t, S_{t}^{j, M}\right)}}
$$

Note that they depend not only on the stocks but also on the index. More importantly, it is commonly observed that the more the market is volatile, the more the stocks tend to be highly correlated. This feature is reproduced here as we can easily check that an increase in the index volatility, with everything else left unchanged, produces an increase in the cross-correlations.

In a recent paper, Cizeau et al. [8] show that it is possible to capture the essential features of stocks crosscorrelations, in particular in extreme market conditions, by a simple non-Gaussian one factor model. The authors successfully compare different empirical measures of correlation with the prediction of the following model :

$$
r_{j}(t)=\beta_{j} r_{I}(t)+\epsilon_{j}(t)
$$

where $r_{j}(t)=\frac{S_{t}^{j}}{S_{t-1}^{j}}-1$ is the daily return of stock $j, r_{I}(t)$ is the daily return of the market and the residuals $\epsilon_{j}(t)$ are independent random variables following a fat-tailed distribution 3 .

\footnotetext{
${ }^{3}$ The authors have chosen a Student distribution in their numerical experiments.
} 
Our model is in line with (4). Indeed, since the beta coefficients are usually narrowly distributed around 1, the factor $\sum_{j=1}^{M} \beta_{j} w_{j} S_{t}^{j, M}$ of $\sigma\left(t, I_{t}^{M}\right)$ in (3) is close to $I_{t}^{M}$. Moreover, since

$$
\mathbb{E}\left(\left(\int_{0}^{T} \sum_{j=1}^{M} w_{j} S_{t}^{j, M} \eta_{j}\left(t, S_{t}^{j, M}\right) d W_{t}^{j}\right)^{2}\right) \leq \sum_{j=1}^{M} w_{j}^{2} \sup _{1 \leq j \leq M} \int_{0}^{T} \mathbb{E}\left(\left(S_{t}^{j, M} \eta_{j}\left(t, S_{t}^{j, M}\right)\right)^{2}\right) d t \sim \sum_{j=1}^{M} w_{j}^{2} T,
$$

one can neglect the term $\sum_{j=1}^{M} w_{j} S_{t}^{j, M} \eta_{j}\left(t, S_{t}^{j, M}\right) d W_{t}^{j}$ in the dynamics of the index when $\sum_{j=1}^{M} w_{j}^{2}$ is small. Of course, this approximation worsens when the maturity $T$ increases. The latter condition is satisfied when $M$ is large and the weighting vector $\left(w_{1}, \ldots, w_{M}\right)$ is close to the vector $\left(\frac{1}{M}, \ldots, \frac{1}{M}\right)$ with constant coefficients for which $\sum_{j=1}^{M} \frac{1}{M^{2}}=\frac{1}{M}$. Then, if we denote by $r_{j}$ the log-return of the stock $j$ and by $r_{I^{M}}$ the log-return of the index, both on a daily basis, we will have

$$
r_{j}=\beta_{j} r_{I^{M}}+\eta_{j} \Delta W^{j}+\mathrm{drift},
$$

where $\Delta W^{j}$ is an independent Gaussian noise. Consequently, in our model too, the return of a stock is decomposed into a systemic part driven by the index, which represents the market, and a residual part.

\section{Asymptotics for a large number of underlying stocks}

The number of underlying components of an index is usually larg t4 As discussed in the previous section, when $\sum_{j=1}^{M} w_{j}^{2}$ is small, one can neglect the term $\sum_{j=1}^{M} w_{j} S_{t}^{j, M} \eta_{j}\left(t, S_{t}^{j, M}\right) d W_{t}^{j}$ in (3) and derive a simplified approximate dynamics for the index. The aim of this section is to quantify the error we commit by doing so.

To be specific, consider the limit candidate $\left(I_{t}\right)_{t \in[0, T]}$ solution of the following SDE :

$$
\left\{\begin{aligned}
d I_{t} & =(r-\delta) I_{t} d t+\beta I_{t} \sigma\left(t, I_{t}\right) d B_{t} \\
I_{0} & =i_{0}
\end{aligned}\right.
$$

where $i_{0}=\sum_{j=1}^{M} w_{j} s_{0}^{j}$ and $\delta$ and $\beta$ are two constant parameters that will be discussed later.

In the following theorem, we give an upper bound for the $L^{2 p}$-distance between $\left(I_{t}^{M}\right)_{t \in[0, T]}$ and $\left(I_{t}\right)_{t \in[0, T]}$ under mild assumption on the volatility coefficients :

Theorem 1 - Let $p \in \mathbb{N}^{*}$. Under assumption (H[1) and if the following assumptions on the volatility coefficients hold,

(H2) $\exists K_{b}$ such that $\forall(t, s) \in[0, T] \times \mathbb{R}_{+}, \quad|\sigma(t, s)|+\left|\eta_{j}(t, s)\right| \leq K_{b}$.

(H3) $\exists K_{\sigma}$ such that $\forall\left(t, s_{1}, s_{2}\right) \in[0, T] \times \mathbb{R}_{+} \times \mathbb{R}_{+}, \quad\left|s_{1} \sigma\left(t, s_{1}\right)-s_{2} \sigma\left(t, s_{2}\right)\right| \leq K_{\sigma}\left|s_{1}-s_{2}\right|$.

then

$$
\mathbb{E}\left(\sup _{0 \leq t \leq T}\left|I_{t}^{M}-I_{t}\right|^{2 p}\right) \leq C_{T}\left(\left(\sum_{j=1}^{M} w_{j}^{2}\right)^{p}+\left(\sum_{j=1}^{M} w_{j}\left|\beta_{j}-\beta\right|\right)^{2 p}+\left(\sum_{j=1}^{M} w_{j}\left|\delta_{j}-\delta\right|\right)^{2 p}\right)
$$

where

$$
C_{T}=8^{2 p-1} T^{p}\left(T^{p}+K_{p} K_{b}^{2 p}\right) C_{p} \exp \left(4^{2 p-1} T\left(2^{2 p-1} K_{p} T^{p-1}\left(\beta K_{\sigma}\right)^{2 p}+(2 T)^{2 p-1} \delta^{2 p}+r^{2 p} T^{2 p-1}\right)\right)
$$

and

$$
C_{p}=\max _{1 \leq j \leq M}\left|s_{0}^{j}\right|^{2 p} \exp \left(\left(2 r+(2 p-1)\left(\max _{j \geq 1} \beta_{j}^{2}+1\right) K_{b}^{2}\right) p T\right) .
$$

\footnotetext{
${ }^{4} 500$ stocks for the S\&P 500 index, 100 stocks for the FTSE 100 index, 40 stocks for the CAC40 index, etc.
} 
According to this result proved in the appendix, the smaller $P_{w}^{M} \stackrel{\text { def }}{=} \sqrt{\sum_{j=1}^{M} w_{j}^{2}}, P_{\beta}^{M} \stackrel{\text { def }}{=} \sum_{j=1}^{M} w_{j}\left|\beta_{j}-\beta\right|$ and $P_{\delta}^{M} \stackrel{\text { def }}{=} \sum_{j=1}^{M} w_{j}\left|\delta_{j}-\delta\right|$, the closer $I$ and $I^{M}$. The first quantity $P_{w}^{M}$ is small when the weighting vector $\left(w_{1}, \ldots, w_{M}\right)$ is close to $\left(\frac{1}{M}, \ldots, \frac{1}{M}\right)$ and $M$ is large. Let us now discuss how to choose $\beta$ and $\delta$ minimizing $P_{\beta}^{M}$ and $P_{\delta}^{M}$. Let $Y_{\beta}$ and $Y_{\delta}$ be discrete random variables having the following probability distributions :

$$
\forall j \in\{1, \ldots, M\}, \quad \mathbb{P}\left(Y_{\beta}=\beta_{j}\right)=\frac{w_{j}}{\sum_{i=1}^{M} w_{i}} \text { and } \mathbb{P}\left(Y_{\delta}=\delta_{j}\right)=\frac{w_{j}}{\sum_{i=1}^{M} w_{i}} .
$$

Then

$$
P_{\beta}^{M}=\left(\sum_{i=1}^{M} w_{i}\right) \times \mathbb{E}\left|Y_{\beta}-\beta\right| \quad \text { and } \quad P_{\delta}^{M}=\left(\sum_{i=1}^{M} w_{i}\right) \times \mathbb{E}\left|Y_{\delta}-\delta\right| .
$$

Consequently, the optimal choice of the parameters is the median 5 of $Y_{\beta}$ for $\beta$ and the median of $Y_{\delta}$ for $\delta$. Nevertheless, to preserve the interpretation of $\beta_{j}$ as $\frac{\operatorname{Cov}\left(r_{j}, r_{I}\right)}{\operatorname{Var}\left(r_{I}\right)}$ which is equal to $\frac{\beta \beta_{j}}{\beta^{2}}$ for the simplified index dynamics, one should take $\beta=1$. In Table 1, we see that on the example of the Eurostoxx index at December 21 2007, the optimal choice of $\beta$ is very close to 1 and that the quantities of interest, $\left(P_{\beta_{o p t}}^{M}\right)^{2}$ and $\left(P_{\beta=1}^{M}\right)^{2}$ are also very close to each other.

\begin{tabular}{|c|c|c|c|}
\hline$\left(P_{w}^{M}\right)^{2}$ & $\beta_{o p t}$ & $\left(P_{\beta_{o p t}}^{M}\right)^{2}$ & $\left(P_{\beta=1}^{M}\right)^{2}$ \\
\hline 0.026 & 0.975 & 0.0173 & 0.0174 \\
\hline
\end{tabular}

Table 1: Computation of $\left(P_{w}^{M}\right)^{2}, \beta_{\text {opt }}$ and $\left(P_{\beta_{o p t}}^{M}\right)^{2}$ for the Eurostoxx index at December 21, 2007. The beta coefficients are estimated on a two year history.

The next theorem states that, under an additional assumption on the volatility coefficients, the $L^{2 p_{-}}$ distance between a stock $\left(S_{t}^{j, M}\right)_{t \in[0, T]}$ and the solution of the SDE obtained by replacing $I^{M}$ by $I$

$$
\frac{d S_{t}^{j}}{S_{t}^{j}}=\left(r-\delta_{j}\right) d t+\beta_{j} \sigma\left(t, I_{t}\right) d B_{t}+\eta_{j}\left(t, S_{t}^{j}\right) d W_{t}^{j}, S_{0}^{j}=s_{0}^{j}
$$

is also controlled by $2 p$-powers of $P_{w}^{M}, P_{\beta}^{M}$ and $P_{\delta}^{M}$. One major drawback of the limiting simplified model (5)(6) is that the limit index $I_{t}$ is only approximately equal to the reconstructed index level $\bar{I}_{t}^{M} \stackrel{\text { def }}{=} \sum_{j=1}^{M} w_{j} S_{t}^{j}$. The next result also gives an estimation of the difference between $I^{M}$ and $\bar{I}^{M}$ in terms of $P_{w}^{M}, P_{\beta}^{M}$ and $P_{\delta}^{M}$, which combined with the previous theorem, provides an estimation of the difference between $I$ and $\bar{I}^{M}$.

Theorem 2 - Let $p \in \mathbb{N}^{*}$. Under the assumptions of Theorem 1 and if

(H4) $\exists K_{\eta}$ such that $\forall j, \forall\left(t, s_{1}, s_{2}\right) \in[0, T] \times \mathbb{R}_{+} \times \mathbb{R}_{+}, \quad\left|s_{1} \eta_{j}\left(t, s_{1}\right)-s_{2} \eta_{j}\left(t, s_{2}\right)\right| \leq K_{\eta}\left|s_{1}-s_{2}\right|$. $\exists K_{\text {Lip }}$ such that $\forall\left(t, s_{1}, s_{2}\right) \in[0, T] \times \mathbb{R}_{+} \times \mathbb{R}_{+}, \quad\left|\sigma\left(t, s_{1}\right)-\sigma\left(t, s_{2}\right)\right| \leq K_{\text {Lip }}\left|s_{1}-s_{2}\right|$.

Then, $\forall j \in\{1, \ldots, M\}$,

$$
\mathbb{E}\left(\sup _{0 \leq t \leq T}\left|S_{t}^{j, M}-S_{t}^{j}\right|^{2 p}\right) \leq \widetilde{C}_{T}^{j}\left(\left(\sum_{j=1}^{M} w_{j}^{2}\right)^{p}+\left(\sum_{j=1}^{M} w_{j}\left|\beta_{j}-\beta\right|\right)^{2 p}+\left(\sum_{j=1}^{M} w_{j}\left|\delta_{j}-\delta\right|\right)^{2 p}\right)
$$

\footnotetext{
${ }^{5}$ The median of a real random variable $X$ is any real number $m$ satisfying :

$$
\mathbb{P}(X \leq m) \geq \frac{1}{2} \text { and } \mathbb{P}(X \geq m) \geq \frac{1}{2} .
$$

It has the property of minimizing the $L^{1}$-distance to $X: m=\arg \min _{x \in \mathbb{R}} \mathbb{E}|X-x|$.
} 
where

$$
\widetilde{C}_{T}^{j}=6^{2 p-1} K_{p} T^{p} \beta_{j}^{2 p} C_{2 p}^{\frac{1}{2}} K_{L i p}^{2 p} e^{3^{2 p-1}\left(\left(r-\delta_{j}\right)^{2 p} T^{2 p-1}+K_{p} T^{p-1} K_{\eta}^{2 p}+2^{2 p-1} K_{p} T^{p-1} \beta_{j}^{2 p} K_{b}^{2 p}\right) T .} .
$$

Moreover, for $\bar{I}_{t}^{M}=\sum_{j=1}^{M} w_{j} S_{t}^{j}$, one has

$$
\mathbb{E}\left(\sup _{0 \leq t \leq T}\left|I_{t}^{M}-\bar{I}_{t}^{M}\right|^{2 p}\right) \leq \widetilde{C}_{T}\left(\sum_{j=1}^{M} w_{j}\right)^{2 p}\left(\left(\sum_{j=1}^{M} w_{j}^{2}\right)^{p}+\left(\sum_{j=1}^{M} w_{j}\left|\beta_{j}-\beta\right|\right)^{2 p}+\left(\sum_{j=1}^{M} w_{j}\left|\delta_{j}-\delta\right|\right)^{2 p}\right)
$$

where $\widetilde{C}_{T}=\max _{1 \leq j \leq M} \widetilde{C}_{T}^{j}$.

The proof can also be found in the appendix. In the following corollary, we consider the limit $M \rightarrow \infty$ supposing that the weight of the $j$-th stock, now denoted by $w_{j}^{M}$, depends on $M$.

Corollary 3 - Under the assumptions of Theorems 1 and 2 and if

(H5) there exists a finite constant $A$ such that $\max _{j \geq 1}\left(\left(s_{0}^{j}\right)^{2}+\left(\beta_{j}\right)^{2}+\left(\delta_{j}\right)^{2}\right) \leq A$,

$$
I_{0}^{M}=\sum_{j=1}^{M} w_{j}^{M} s_{0}^{j} \underset{M \rightarrow \infty}{\longrightarrow} i_{0} \in(0,+\infty)
$$

$$
P_{w}^{M}=\sqrt{\sum_{j=1}^{M}\left(w_{j}^{M}\right)^{2}} \underset{M \rightarrow \infty}{\longrightarrow} 0,
$$

(H8) $P_{\beta}^{M}=\sum_{j=1}^{M} w_{j}^{M}\left|\beta_{j}-\beta\right| \underset{M \rightarrow \infty}{\longrightarrow} 0$,

(H) $\quad P_{\delta}^{M}=\sum_{j=1}^{M} w_{j}^{M}\left|\delta_{j}-\delta\right| \underset{M \rightarrow \infty}{\longrightarrow} 0$

then, for any $p \in \mathbb{N}^{*}$, one has

$$
\mathbb{E}\left(\sup _{0 \leq t \leq T}\left|I_{t}^{M}-I_{t}\right|^{2 p}\right) \underset{M \rightarrow \infty}{\longrightarrow} 0 \text { and } \forall j \in \mathbb{N}^{*}, \quad \mathbb{E}\left(\sup _{0 \leq t \leq T}\left|S_{t}^{j, M}-S_{t}^{j}\right|^{2 p}\right) \underset{M \rightarrow \infty}{\longrightarrow} 0 .
$$

If, in addition, $\sup _{M} \sum_{j=1}^{M} w_{j}^{M}<\infty$, then $\mathbb{E}\left(\sup _{0 \leq t \leq T}\left|I_{t}^{M}-\bar{I}_{t}^{M}\right|^{2 p}\right) \longrightarrow_{M \rightarrow \infty} 0$.

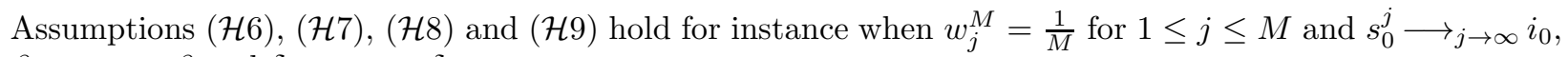
$\beta_{j} \longrightarrow_{j \rightarrow \infty} \beta$ and $\delta_{j} \longrightarrow_{j \rightarrow \infty} \delta$. 


\section{Simplified model}

To sum up, we have shown that, under mild assumptions, when the number of underlying stocks is large, the original model may be approximated by the following dynamics

$$
\begin{aligned}
\forall j \in\{1, \ldots, M\}, \quad \frac{d S_{t}^{j}}{S_{t}^{j}} & =\left(r-\delta_{j}\right) d t+\beta_{j} \sigma\left(t, I_{t}\right) d B_{t}+\eta_{j}\left(t, S_{t}^{j}\right) d W_{t}^{j} \\
\frac{d I_{t}}{I_{t}} & =\left(r-\delta_{I}\right) d t+\sigma\left(t, I_{t}\right) d B_{t} .
\end{aligned}
$$

Of course the distance between this limiting model and the original one increases with the maturity.

Interestingly, we end up with a local volatility model for the index and, for each stock, a stochastic volatility model decomposed into a systemic part driven by the index level and an intrinsic part. The calibration procedures presented in the next section are based on this intuition : even in the original model, we are going to calibrate $\sigma$ as if it was the local volatility function of the index.

Note that this simplified model is not valid for options written on the index together with all its composing stocks since the index is no longer an exact, but an approximate, weighted sum of the stocks. In this case, one should consider the reconstructed index $\bar{I}_{t}^{M}=\sum_{j=1}^{M} w_{j} S_{t}^{j}$ or use the original model. The simplified model can be used for options written on the stocks or on the index or even on the index together with few stocks.

\section{Model calibration}

Calibration, which is how to determine the model parameters in order to fit market prices at best, is of paramount importance in practice. In the following, we try to tackle this issue for both our simplified and original models.

\subsection{Simplified model}

In the simplified limiting model, the only factor which influences the dynamics of a given stock is the simplified index $I_{t}$ which evolves according to an autonomous SDE. So it is enough to address the calibration of a given stock together with the index and we drop the index $j$ of the stock for notational simplicity.

$$
\begin{aligned}
& \frac{d S_{t}}{S_{t}}=(r-\delta) d t+\beta \sigma\left(t, I_{t}\right) d B_{t}+\eta\left(t, S_{t}\right) d W_{t}, S_{0}=s_{0} \\
& \frac{d I_{t}}{I_{t}}=\left(r-\delta_{I}\right) d t+\sigma\left(t, I_{t}\right) d B_{t}, I_{0}=i_{0} .
\end{aligned}
$$

The short interest rate and the dividend yields can be extracted from the market. The calibration of the local volatility function $\sigma$ to fit index option prices is a classical problem. According to Dupire [10, if $C_{I}(t, K)$ denotes the market price of the call option with maturity $t$ and strike $K$ written on the index, then for

$$
\sigma^{2}(t, K)=2 \frac{\frac{\partial C_{I}}{\partial t}(t, K)+\left(r-\delta_{I}\right) K \frac{\partial C_{I}}{\partial K}(t, K)+\delta_{I} C_{I}(t, K)}{K^{2} \frac{\partial^{2} C_{I}}{\partial K^{2}}(t, K)},
$$

one has $C_{I}(t, K)=\mathbb{E}\left(e^{-r t}\left(I_{t}-K\right)^{+}\right)$for all $t, K>0$. Of course, in practice the market quotes call options only for a finite number of couples $(t, K)$. What seems a common practice among banks is to look for $\sigma$ in a parametric family of functions and compute the parameters minimizing the distance between these quoted prices and the call prices associated with the parametrized local volatility function. Since each practitioner may choose his favorite procedure to address this classical problem of local volatility calibration, we will not enter in more details. 
We also assume that a local volatility function is associated with the stock by the same procedure and denote by $v_{l o c}(t, x)$ the local variance function of the stock computed as the square of this local volatility function. So the local volatility model

$$
\frac{d \bar{S}_{t}}{\bar{S}_{t}}=(r-\delta) d t+\sqrt{v_{l o c}\left(t, \bar{S}_{t}\right)} d W_{t}, \bar{S}_{0}=s_{0}
$$

is calibrated to the quoted prices of vanilla options written on the stock. In (8), by independence between $B$ and $W$, the variance of the stock at time $t$ is equal to $\beta^{2} \sigma^{2}\left(t, I_{t}\right)+\eta^{2}\left(t, S_{t}\right)$. According to Gyöngy [11, if

$$
\forall t, x>0, \mathbb{E}\left(\beta^{2} \sigma^{2}\left(t, I_{t}\right)+\eta^{2}\left(t, S_{t}\right) \mid S_{t}=x\right)=v_{l o c}(t, x)
$$

then (8) and the local volatility model (9) induce the same marginal distributions for the stock and therefore the same prices for the vanilla call options written on it : $\mathbb{E}\left(e^{-r t}\left(S_{t}-K\right)^{+}\right)=\mathbb{E}\left(e^{-r t}\left(\bar{S}_{t}-K\right)^{+}\right)$for all $t, K>0$. Hence if

$$
\forall t, x>0, \eta(t, x)=\sqrt{v_{l o c}(t, x)-\beta^{2} \mathbb{E}\left(\sigma^{2}\left(t, I_{t}\right) \mid S_{t}=x\right)},
$$

then the stock dynamics in (8) is calibrated to the quoted prices of the vanilla options written on the stock. It remains to choose the coefficient $\beta$ and the function $\eta$ so that this equality is satisfied. The fact that the law of $\left(S_{t}, I_{t}\right)$ given by (8) and therefore the conditional expectation in (10) depend on $(\beta, \eta)$ makes this problem difficult. Nevertheless, intuitively, when one fixes a value of $\beta$ that is not too large, one should be able to find a function $\eta$ such that (10) is satisfied. The calibration of the stock smile seems over-parametrized and one should rely on the interpretation of $\beta$ as a regression coefficient to choose its value. This issue is discussed in the next section. Then we explain how to approximate the conditional expectation and deduce $\eta$ for a fixed value of $\beta$.

Let us already point out that the calibration of our simplified model gives an advantage to the fit of index option prices in comparison with options written on the stocks, which is in line with the market since index options are usually very liquid in comparison with individual stock options.

\subsubsection{Choice of the coefficient $\beta$}

The interpretation of $\beta$ as the regression coefficient of the log-returns of the stock with respect to the log-returns of the index makes it possible to estimate this coefficient on historical data. Nevertheless, when the historical estimator $\beta_{\text {hist }}$ is large, then the difference in the r.h.s. of (10) may become negative for some $(t, x)$ when $\beta=\beta_{\text {hist }}$. Then the square root is no longer defined and calibration for this choice of $\beta$ is no longer possible.

In Figure 1, we have plotted the local volatility of the stock $x \mapsto \sqrt{v_{l o c}\left(T, s_{0} x\right)}$, the local volatility of the index $x \mapsto \sigma\left(T, i_{0} x\right)$, the systemic part of the volatility of the stock $x \mapsto \beta_{\text {hist }} \sigma\left(T, s_{0} x\right)$ and $x \mapsto$ $\beta_{\text {hist }} \sqrt{\mathbb{E}\left(\sigma^{2}\left(T, I_{T}\right) \mid S_{T}=s_{0} x\right)}$ when $\eta$ is set to zero (which intuitively gives the lowest local volatility function of the stock that one can obtain in our model (8)) as functions of the moneyness for a maturity $T=1$ year. We considered three representative components of the Eurostoxx which is composed of $M=50$ stocks : AXA, ALCATEL and CARREFOUR at December 21, 2007. We made this choice deliberately in order to point out the extreme situations that one can face :

- AXA is an example of a stock with a high historical beta coefficient $\left(\beta_{\text {hist }}=1.4\right)$,

- CARREFOUR is an example of a stock with a low historical beta coefficient $\left(\beta_{\text {hist }}=0.7\right)$,

- ALCATEL is an example of a stock with a high volatility level but with a rather flat smile $\left(\beta_{\text {hist }}=1.1\right)$.

Clearly, we can deduce that the market is choosing a $\beta$ coefficient for both AXA and ALCATEL that is lower than the historical one whereas, for CARREFOUR, one can plug the historical $\beta$, or even a larger one, in (8) and still be able to calibrate the model. 


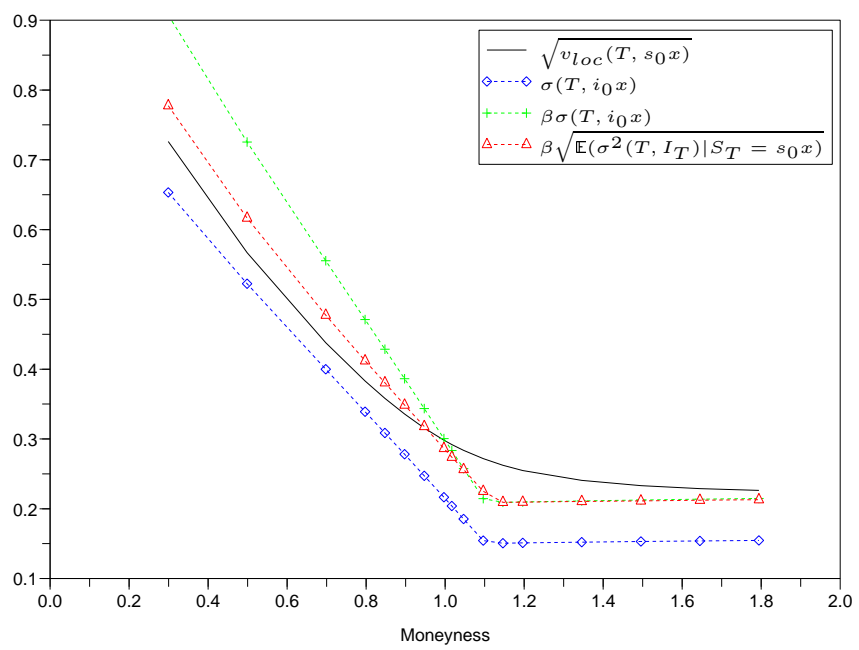

ALCATEL

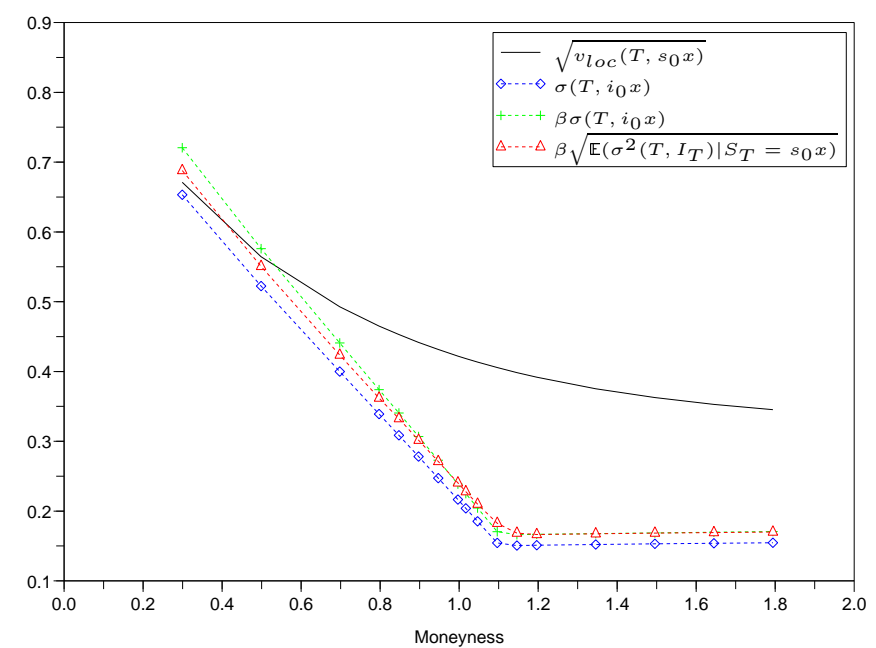

CARREFOUR

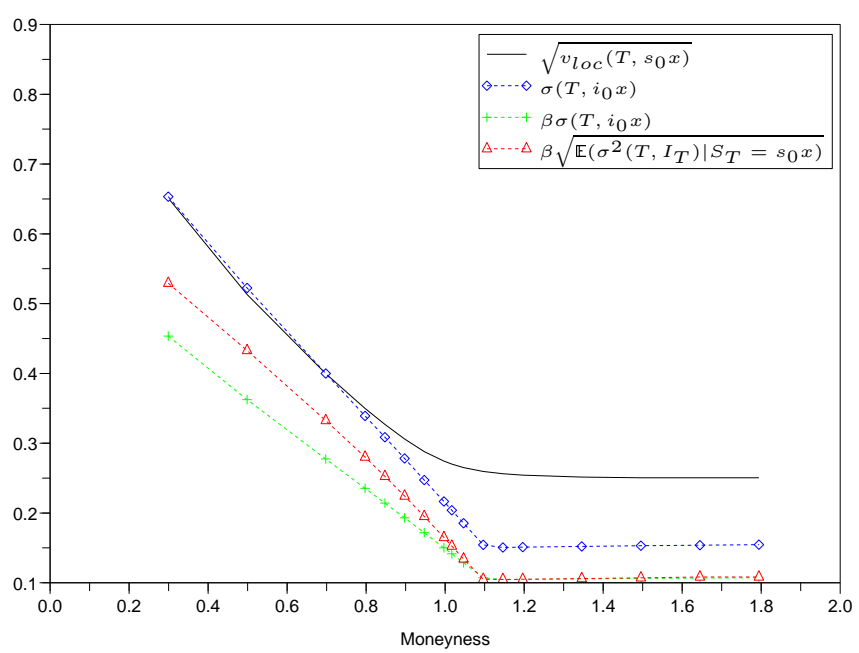

Figure 1: Local volatility $x \mapsto \sqrt{v_{\text {loc }}\left(T, s_{0} x\right)}$ together with $x \mapsto \sigma\left(T, i_{0} x\right), x \mapsto \beta_{\text {hist }} \sigma\left(T, i_{0} x\right)$ and $x \mapsto$ $\beta_{\text {hist }} \sqrt{\mathbb{E}\left(\sigma^{2}\left(T, I_{T}\right) \mid S_{T}=s_{0} x\right)}$ when $\eta$ is set to zero for AXA, ALCATEL and CARREFOUR. 
A satisfactory way to handle the estimation of the beta coefficient would be to compute an implied beta calibrated to the prices of options involving the correlation between the stock and the index. Unfortunately, no such option is liquid in the market (the most liquid correlation swaps are sensitive to an average correlation between all the stocks composing the index).

So we suggest to choose

$$
\beta=\min \left(\beta_{h i s t}, \inf _{t, x>0} \frac{\sqrt{v_{l o c}\left(t, s_{0} x\right)}}{\sigma\left(t, i_{0} x\right)}\right) .
$$

Even if we have no proof that this choice of beta makes the calibration possible, it is sensible and we have checked that it works on the three examples of AXA, ALCATEL and CARREFOUR.

When one is interested in options written on the index together with all its components, one should use the reconstructed index level $\bar{I}_{t}^{M}=\sum_{j=1}^{M} w_{j} S_{t}^{j}$ instead of $I_{t}$. Of course, the reconstructed index dynamics

will reproduce the quoted prices of vanilla options written on the index all the better as $\bar{I}_{t}^{M}$ is close to the calibrated limiting index level $I_{t}$. According to Theorems 1 and 2, for this latter property to hold, one needs $P_{\beta=1}^{M}=\sum_{j=1}^{M} w_{j}\left|\beta_{j}-1\right| \geq\left|\sum_{j=1}^{M} w_{j} \beta_{j}-\sum_{j=1}^{M} w_{j}\right|$ to be small. When, because of the minimum in equality (11), $P_{\beta=1}^{M}$ is larger for the actual choice of coefficients $\beta$ than for the historical choice, one may take larger values of beta for stocks like CARREFOUR to decrease $P_{\beta=1}^{M}$ and improve the calibration of the reconstructed index.

\subsubsection{Estimation of the conditional expectation}

The idea behind the following techniques is to circumvent the difficulty of calibrating the volatility coefficient $\eta$. Indeed, if we plug the formula (10) in (8), we obtain a stochastic differential equation that is nonlinear in the sense of McKean :

$$
\begin{aligned}
& \frac{d S_{t}}{S_{t}}=(r-\delta) d t+\beta \sigma\left(t, I_{t}\right) d B_{t}+\sqrt{v_{l o c}\left(t, S_{t}\right)-\beta^{2} \mathbb{E}\left(\sigma^{2}\left(t, I_{t}\right) \mid S_{t}\right)} d W_{t}, S_{0}=s_{0} \\
& \frac{d I_{t}}{I_{t}}=\left(r-\delta_{I}\right) d t+\sigma\left(t, I_{t}\right) d B_{t}, I_{0}=i_{0} .
\end{aligned}
$$

For an introduction to the stochastic differential equations nonlinear in the sense of McKean and to propagation of chaos, we refer to the lecture notes of Sznitman [18] and Méléard [15]. In our case, the nonlinearity appears in the diffusion coefficient through the conditional expectation term. This makes the natural question of existence and uniqueness of a solution very difficult to handle. The case of a drift coefficient involving a conditional expectation has only been handled recently even for a constant diffusion coefficient (see for instance Talay and Vaillant [19] and Dermoune [9]). Meanwhile, it is possible to simulate such a stochastic differential equation by means of a system of $N$ interacting paths using either a non-parametric estimation of the conditional expectation or regression techniques. The advantage of the regression approach over the non-parametric estimation is that it also yields a smooth approximation of the function $x \mapsto \mathbb{E}\left(\sigma^{2}\left(t, I_{t}\right) \mid S_{t}=x\right)$ whereas, with a non-parametric method, one has to interpolate the estimated function and to carefully tune the window parameter to obtain a smooth approximation.

\subsection{2a Non-parametric estimation}

Non-parametric estimators of the conditional expectation, and more generally non-parametric density estimators, have been widely studied in the literature. We will focus on kernel estimators of the NadarayaWatson type (see [20] and [16]) : given $N$ observations $\left(S_{i, t}, I_{i, t}\right)_{i=1 \ldots N}$ of $\left(S_{t}, I_{t}\right)$, we consider the kernel conditional expectation estimator of $\mathbb{E}\left(\sigma^{2}\left(t, I_{t}\right) \mid S_{t}=x\right)$ given by 


$$
\frac{\sum_{i=1}^{N} \sigma^{2}\left(t, I_{i, t}\right) K\left(\frac{x-S_{i, t}}{h_{N}}\right)}{\sum_{i=1}^{N} K\left(\frac{x-S_{i, t}}{h_{N}}\right)}
$$

where $K$ is a non-negative kernel such that $\int_{\mathbb{R}} K(x) d x=1$ and $h_{N}$ is a smoothing parameter which tends to zero as $N \rightarrow+\infty$. This leads to the following system with $N$ interacting particles : $\forall 1 \leq i \leq N$,

$$
\left\{\begin{array}{l}
\frac{d S_{i, N, t}}{S_{i, N, t}}=(r-\delta) d t+\beta \sigma\left(t, I_{i, t}\right) d B_{i, t}+\sqrt{v_{l o c}\left(t, S_{i, N, t}\right)-\beta^{2} \frac{\sum_{j=1}^{N} \sigma^{2}\left(t, I_{j, t}\right) K\left(\frac{S_{i, N, t}-S_{j, N, t}}{h_{N}}\right)}{\sum_{j=1}^{N} K\left(\frac{S_{i, N, t}-S_{j, N, t}}{h_{N}}\right)}} d W_{i, t}, S_{i, N, 0}=s_{0} \\
\frac{d I_{i, t}}{I_{i, t}}=\left(r-\delta_{I}\right) d t+\sigma\left(t, I_{i, t}\right) d B_{i, t}, I_{i, 0}=i_{0}
\end{array}\right.
$$

where $\left(B_{i}, W_{i}\right)_{i \geq 1}$ is a sequence of independent two-dimensional Brownian motions. The integer $i$ indexes the sample-paths of the fixed stock that we consider. In their dynamics, the conditional expectation term has been replaced by interaction. The price in the calibrated model of a European option with maturity $T$ and payoff function $h: C([0, T], \mathbb{R}) \rightarrow \mathbb{R}$ written on the stock may be approximated by

$$
\frac{1}{N} \sum_{i=1}^{N} e^{-r T} h\left(S_{i, N, .}\right)
$$

The $2 N$-dimensional SDE may be discretized using the Euler scheme. Let $n \in \mathbb{N}^{*}$ and $0=t_{0}<\cdots<$ $t_{n}=T$ be the subdivision with step $\frac{T}{n}$ of $[0, T]$. For each $k \in\{0, \ldots, n-1\}, \forall 1 \leq i \leq N$,

$$
\left\{\begin{array}{c}
\bar{S}_{i, N, t_{k+1}}=\bar{S}_{i, N, t_{k}}\left(1+\sqrt{v_{l o c}\left(t_{k}, \bar{S}_{i, N, t_{k}}\right)-\beta^{2} \frac{\sum_{j=1}^{N} \sigma^{2}\left(t_{k}, \bar{I}_{j, t_{k}}\right) K\left(\frac{\bar{S}_{i, N, t_{k}}-\bar{S}_{j, N, t_{k}}}{h_{N}}\right)}{\sum_{j=1}^{N} K\left(\frac{\bar{S}_{i, N, t_{k}}-\bar{S}_{j, N, t_{k}}}{h_{N}}\right)}} \sqrt{\frac{T}{n}} \tilde{G}_{i, k}\right. \\
\left.+(r-\delta) \frac{T}{n}+\beta \sigma\left(t_{k}, \bar{I}_{i, t_{k}}\right) \sqrt{\frac{T}{n}} G_{i, k}\right) \\
\bar{I}_{i, t_{k+1}}=\bar{I}_{i, t_{k}}\left(1+\left(r-\delta_{I}\right) \frac{T}{n}+\sigma\left(t_{k}, \bar{I}_{i, t_{k}}\right) \sqrt{\frac{T}{n}} G_{i, k}\right)
\end{array}\right.
$$

where $\left(G_{i, k}\right)_{1 \leq i \leq N, 0 \leq k \leq n-1}$ and $\left(\tilde{G}_{i, k}\right)_{1 \leq i \leq N, 0 \leq k \leq n-1}$ are independent centered and reduced Gaussian random variables.

\subsection{2b Parametric estimation}

Another approach to estimate conditional expectations is to use parametric estimators, or projection. This idea has also been widely used and studied previously (for example in finance, one can think of the Longstaff-Schwartz algorithm for pricing American options [14]). Noting that the conditional expectation is a projection operator on the space of square integrable random variables, one can approximate $\mathbb{E}\left(\sigma^{2}\left(t, I_{t}\right) \mid S_{t}=x\right)$ by the parametric estimator $\sum_{l=1}^{L} \alpha_{l} f_{l}(x)$ where $\left(f_{l}\right)_{l=1 \ldots L}$ is a functional basis and $\alpha=\left(\alpha_{l}\right)_{l=1 \ldots L}$ is a vector of parameters estimated by least mean squares : given $N$ observations $\left(S_{i, t}, I_{i, t}\right)_{i=1 \ldots N}$ of $\left(S_{t}, I_{t}\right), \alpha$ minimizes $\sum_{i=1}^{N}\left(\sigma^{2}\left(t, I_{i, t}\right)-\sum_{l=1}^{L} \alpha_{l} f_{l}\left(S_{i, t}\right)\right)^{2}$.

\subsubsection{Numerical results}

\subsection{3a A toy example}

We try to calibrate a stock with a local variance function $v_{l o c}$ constant and equal to $v$. We choose $\sigma$ as the local volatility function of the Eurostoxx index fitted to the market at December 21, 2007. 
We simulate the system of $N$ interacting paths (13) and price call options for different strikes using (14). In Figure 2, we plot the implied volatility at $T=1$ obtained for independent simulations of $N=5000$ paths and see that they are indeed close to the desired volatility level $\sqrt{v}$. This example was generated with the following arbitrary set of parameters :

$$
S_{0}=100, \beta=0.7, r=0.05, \delta=\delta_{I}=0, \sqrt{v}=0.6, N=5000, n=20 .
$$

In this example and for all the following numerical experiments, we use a Gaussian kernel : $K(u)=$ $\frac{1}{\sqrt{2 \pi}} e^{-\frac{u^{2}}{2}}$. The smoothing parameter $h_{N}$ is set to $N^{-\frac{1}{5}}$ which is the optimal bandwidth that one obtains when minimizing the asymptotic mean square error of the Nadaraya-Watson estimator under some regularity assumptions and assuming independence of the random variables involved (see for example Bosq [6]).

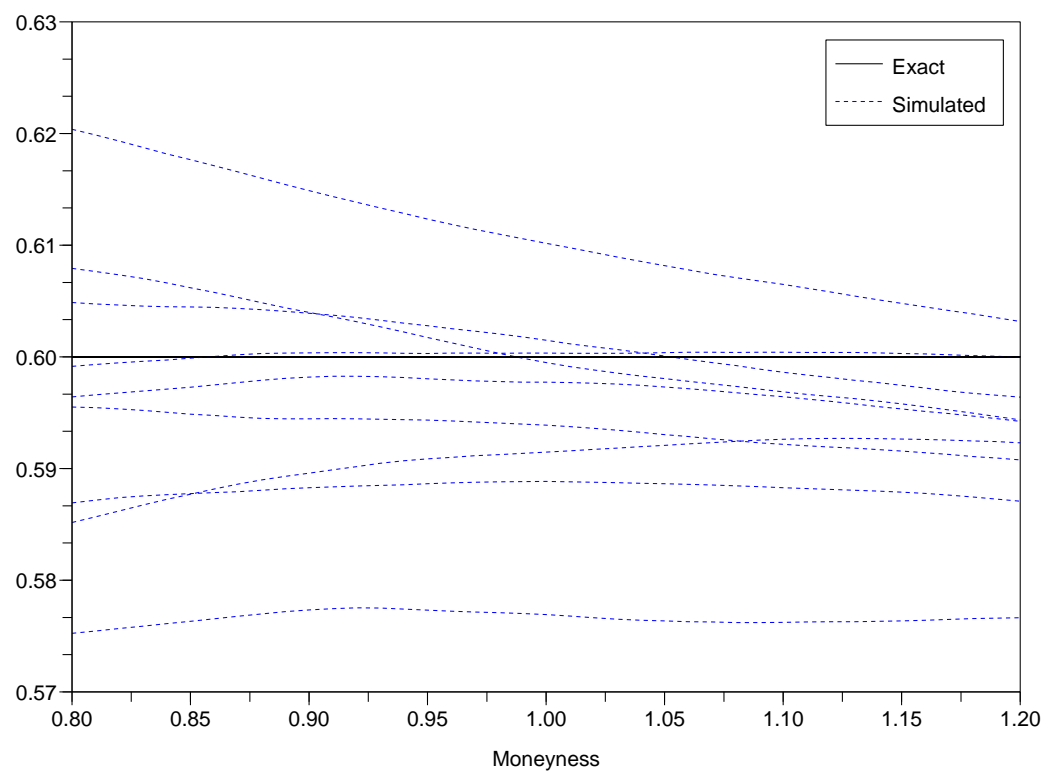

Figure 2: Implied volatility obtained for nine independent simulations with $N=5000$ paths.

\subsection{3b An example with real data}

In the following, we test our model with real data. More precisely, given the local volatilities of the Eurostoxx index and of Carrefour at December 21, 2007, we simulate (12) by different methods for a one year maturity.

\section{An acceleration technique}

The simulation of the particle system is very time consuming : for each discretization step and for each stock particle, one has to make $N$ computations which yield a global complexity of order $O\left(n N^{2}\right)$ where $n$ is the number of time steps in the Euler scheme. Acceleration techniques are thus desirable. One possible method consists in reducing the number of interactions : instead of making $N$ computations for each estimation of the conditional expectation, one can neglect interactions which involve particles which are far away from each other. When the kernel used is non increasing with the absolute value of its argument, the easiest way to implement this idea is to sort the particles at each step and, whenever a contribution of a particle is lower than some fixed threshold, to stop the estimation of the conditional expectation. 
Of course, by doing this, we lose in precision for the same number of interacting particles, especially for deep in/out of the money strikes. But what we gain in terms of computation time is much more important : in Figure 3 we plot the implied volatility obtained by the naive method and the method with the above acceleration technique for the same number $N=10000$ of particles. We take as threshold $\frac{1}{N}$ and set $h_{N}=N^{-\frac{1}{10}}$ for the bandwidth parameter $\sqrt{6}$ and $n=20$ for the number of time steps in the Euler scheme. The computation time, on a computer with a $2.8 \mathrm{Ghz}$ Intel Penthium 4 processor, is of 52 minutes for the naive method and of 5 minutes for the accelerated one.

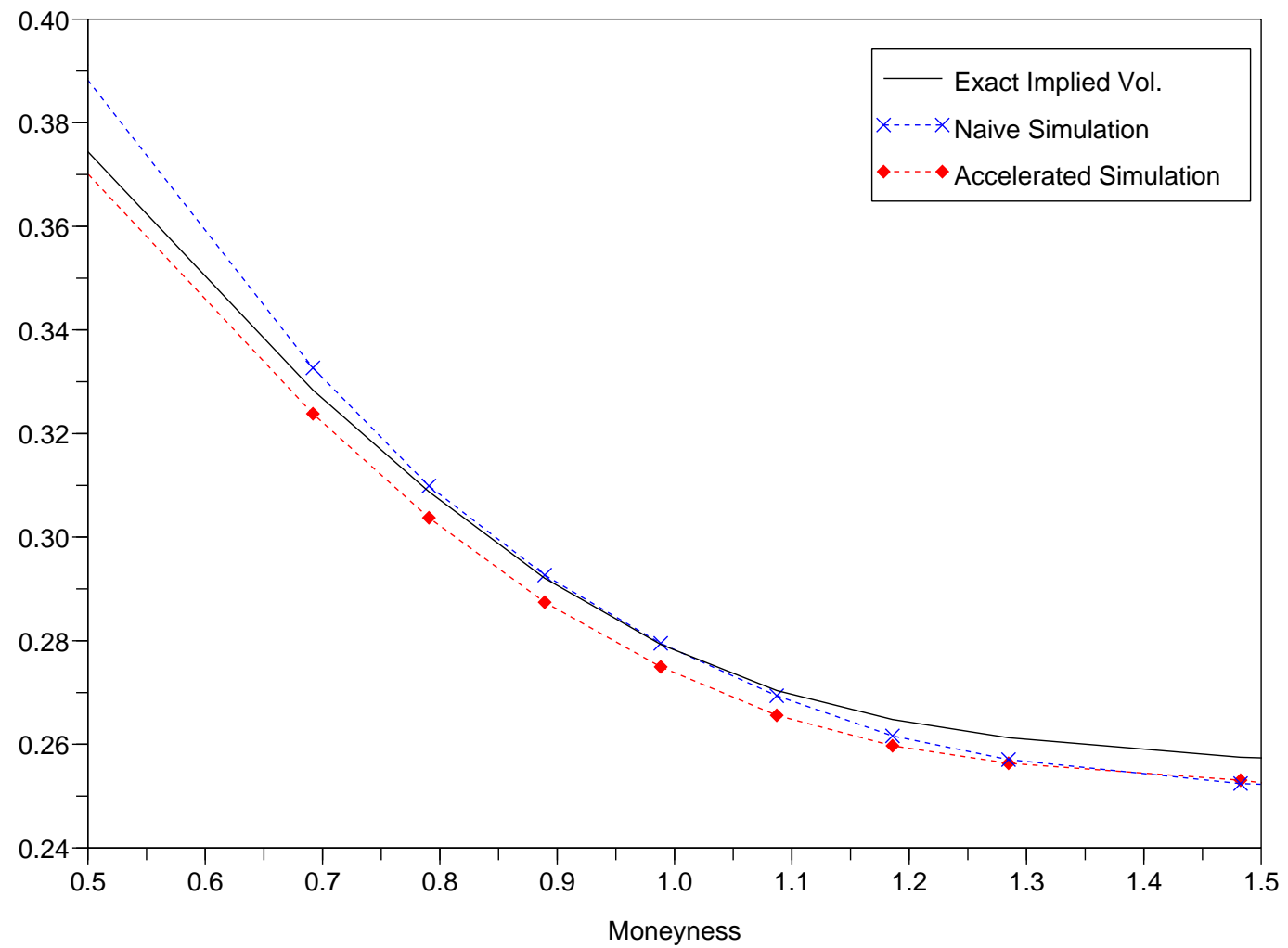

Figure 3: Comparison between the naive technique and the accelerated one for the same number $N=10000$ of particles.

More importantly, we see that the implied volatility $\widehat{\sigma}_{N}$ obtained by simulation of the system with $N$ interacting particles converges to the exact implied volatility $\widehat{\sigma}_{\text {exact }}$ computed from quoted option prices as $N$ tends to $\infty$ : see Figure 4 and Table 2. With a reasonable number of simulated paths, $N=200000$, the error on the implied volatility remains clearly tolerable for practitioners (of the order of $10 \mathrm{bp}$ ) except for a deep in the money call $\left(K=0.3 S_{0}\right)$ where it attains $195 \mathrm{bp}$.

\footnotetext{
${ }^{6}$ In order to smooth the estimation, one has to choose a bandwidth parameter that is greater than the theoretical optimal parameter $N^{-\frac{1}{5}}$.
} 


\begin{tabular}{|c|c|c|c|c|c|c|c|c|c|c|c|}
\hline Moneyness $\left(\frac{K}{S_{0}}\right)$ & 0.30 & 0.49 & 0.69 & 0.79 & 0.89 & 0.99 & 1.09 & 1.19 & 1.28 & 1.48 & 1.98 \\
\hline Error $:\left|\widehat{\sigma}_{N}-\widehat{\sigma}_{\text {exact }}\right|$ & 195 & 36 & 8 & 5 & 2 & 1 & 2 & 9 & 17 & 32 & 56 \\
\hline
\end{tabular}

Table 2: Error (in bp) on the implied volatility with $N=200000$ particles.

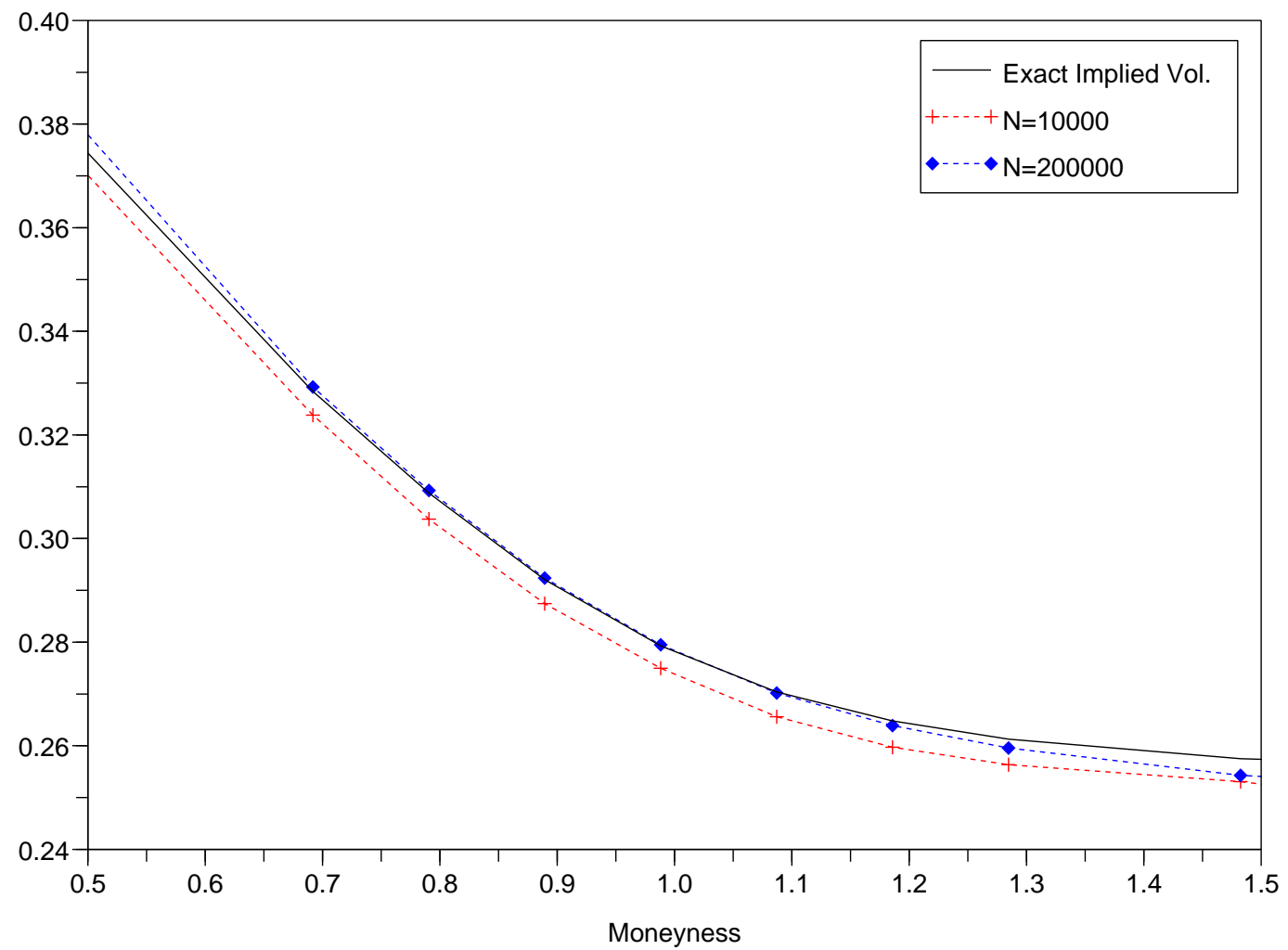

Figure 4: Convergence of the implied volatility obtained with non-parametric estimation as $N \rightarrow+\infty$.

\section{Independent particles}

Unlike the parametric method, non-parametric estimation of the conditional expectation gives the value of the intrinsic volatility $\eta$ at the simulated points only. However, using an interpolation technique, one can first reconstruct $\eta$ with $N_{1}$ dependent particles and then simulate $N_{2}$ independent paths of the 2-dimensional stochastic differential equation (8). By doing so, we speed up the simulations but one has to choose carefully the size $N_{1}$ of the particle system in order to have a reasonable estimation of the intrinsic volatility and to tune the bandwidth parameter in order to smooth the estimation (our numerical tests were done with $N_{1}=1000, N_{2}=100000$ and $\left.h_{N_{1}}=N_{1}^{-\frac{1}{10}}\right)$. In Figures 5 and 6 , we plot the local volatility function $\sqrt{v_{l o c}(t, x)}$ and the intrinsic volatility function $\eta(t, x)$ of the stock. This latter is used to draw independent simulations of the index along with the stock and we see in Figure 7 that the implied volatility obtained is close to the right one, especially near the money. 


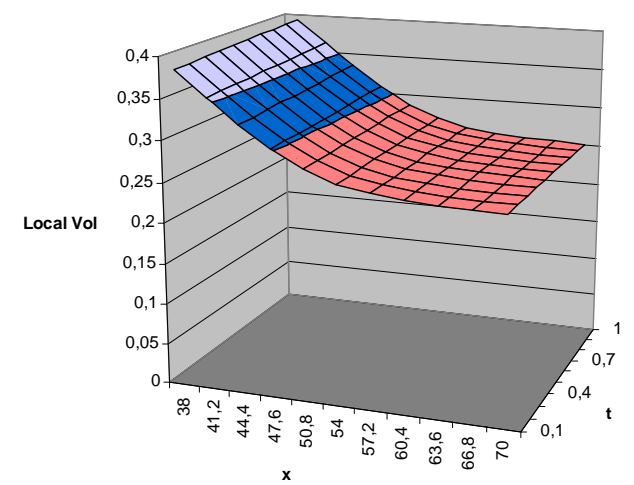

Figure 5: Local volatility function $\sqrt{v_{l o c}(t, x)}$ of the stock.

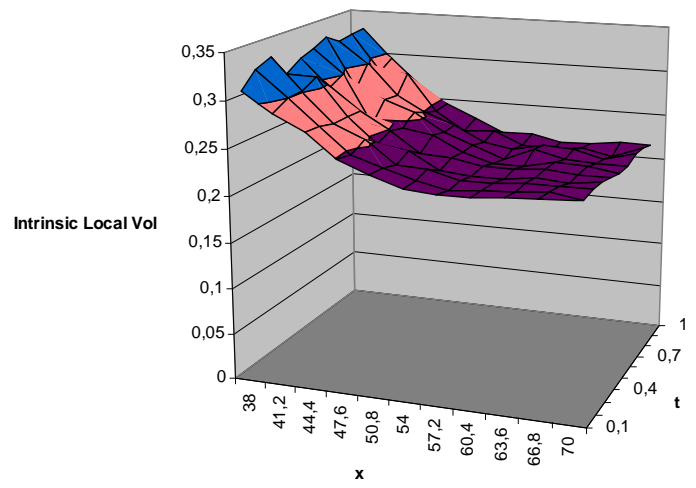

Figure 6: Intrinsic volatility function $\eta(t, x)$ of the stock.

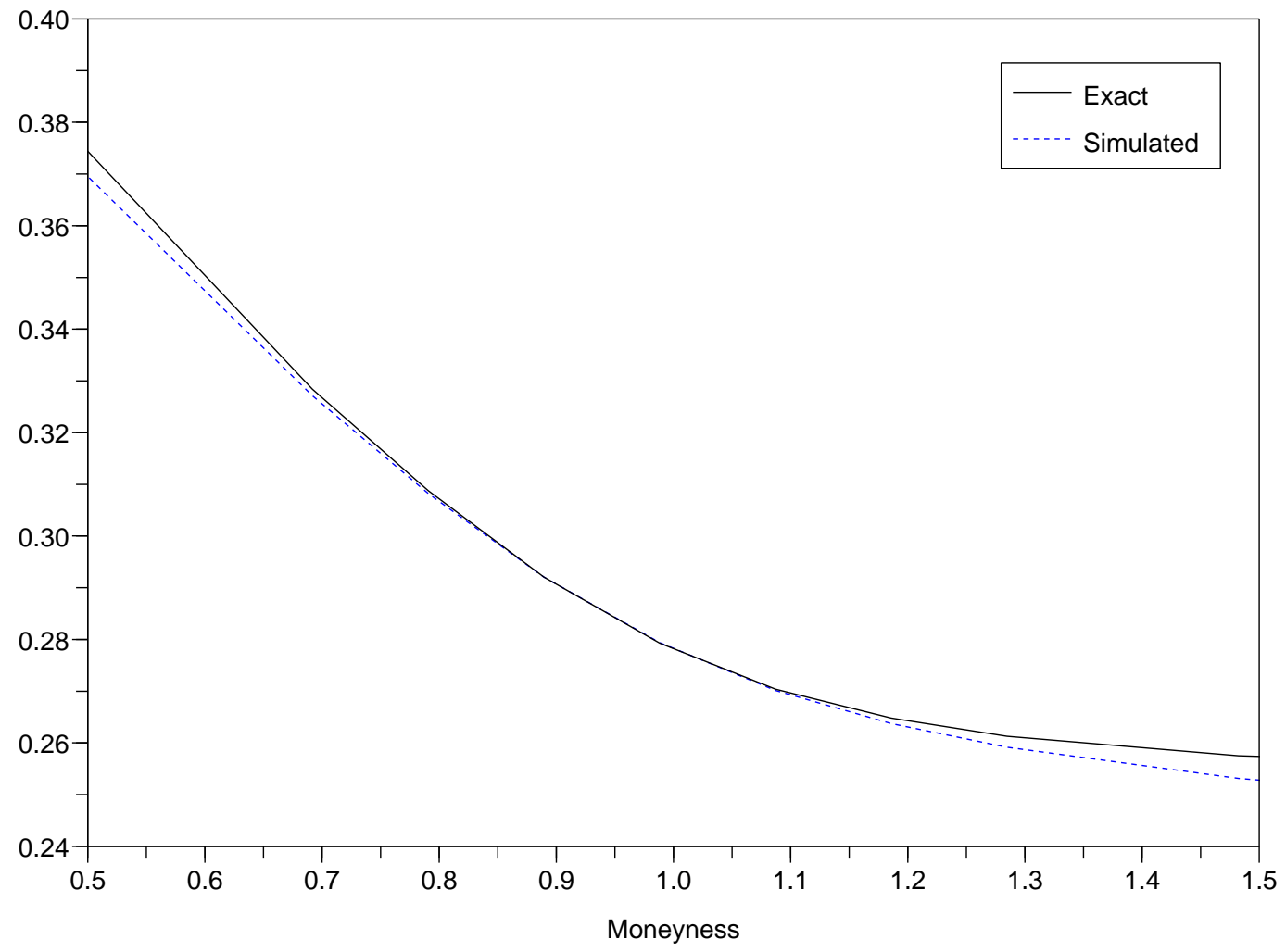

Figure 7: Simulated implied volatility with independent draws. 


\subsection{Original model}

We now turn to the calibration of our original model :

$$
\forall j \in\{1, \ldots, M\}, \quad \frac{d S_{t}^{j, M}}{S_{t}^{j, M}}=\left(r-\delta_{j}\right) d t+\beta_{j} \sigma\left(t, I_{t}^{M}\right) d B_{t}+\eta_{j}\left(t, S_{t}^{j, M}\right) d W_{t}^{j} \text { with } I_{t}^{M}=\sum_{i=1}^{M} w_{i} S_{t}^{i, M}
$$

It is rather complicated to have a perfect calibration for both index and stocks within this framework. Nevertheless, Theorem 1 ensures that the error of calibration of the index smile is small (at least when the maturity is not too large) when $\sigma$ is chosen as a local volatility function fitted to this smile. We also suppose that a local volatility function $\sqrt{v_{l o c}^{j}}$ has been fitted to the market smile of each stock $j$. For the choice of the coef-

ficients $\beta_{j}$, we proceed like in Section 3.1.1. The coefficients $\eta_{j}(t, x)=\sqrt{v_{l o c}^{j}(t, x)-\beta_{j}^{2} \mathbb{E}\left(\sigma^{2}\left(t, I_{t}^{M}\right) \mid S_{t}^{j, M}=x\right)}$ are then calibrated all at the same time using an adaptation of the non-parametric method presented above based on the simulation of $N$ interacting $(M+1)$-dimensional paths.

In comparison with the simplified model, we introduce in the calibration of the index a small error which grows with the maturity $T$. But we guarantee the additivity constraint $I_{t}^{M}=\sum_{i=1}^{M} w_{i} S_{t}^{i, M}$. Note that a similar error spoils the calibration of the reconstructed index in the simplified model (see the discussion at the end of Section 3.1.1).

In what follows, we illustrate the effect of Theorems 1 and 2 and compare our models with a constant correlation model.

\section{Illustration of Theorems 1 and 2 and comparison with a constant correlation model}

The objective of this section is to compare index and individual stock smiles obtained with three different models : our original model (15), the simplified one (after letting $M \rightarrow \infty$ ) and a model with constant correlation coefficient. More precisely, we consider the following dynamics

1. The original model

$$
\forall j \in\{1, \ldots, M\}, \quad \frac{d S_{t}^{j, M}}{S_{t}^{j, M}}=r d t+\sigma\left(t, I_{t}^{M}\right) d B_{t}+\eta\left(t, S_{t}^{j, M}\right) d W_{t}^{j} \text { with } I_{t}^{M}=\sum_{i=1}^{M} w_{i} S_{t}^{i, M} .
$$

2. The simplified model

$$
\begin{aligned}
\forall j \in\{1, \ldots, M\}, \quad \frac{d S_{t}^{j}}{S_{t}^{j}} & =r d t+\sigma\left(t, I_{t}\right) d B_{t}+\eta\left(t, S_{t}^{j}\right) d W_{t}^{j} \\
\frac{d I_{t}}{I_{t}} & =r d t+\sigma\left(t, I_{t}\right) d B_{t} .
\end{aligned}
$$

Here we can also compute the reconstructed index $\bar{I}_{t}^{M}=\sum_{i=1}^{M} w_{i} S_{t}^{i}$.

3. The "market" model

$$
\forall j \in\{1, \ldots, M\}, \frac{d S_{t}^{j}}{S_{t}^{j}}=r d t+\sqrt{v_{l o c}\left(t, S_{t}^{j}\right)} d \widetilde{W}_{t}^{j}
$$

with, $\forall i \neq j, d<\widetilde{W}^{i}, \widetilde{W}^{j}>_{t}=\rho d t$. 
We deliberately dropped the dividend yields and the beta coefficients in order to simplify the numerical experiment. For the function $\sigma$, we take as previously the calibrated local volatility of the Eurostoxx. For $\eta$, which does not depend on $j$, we choose an arbitrary function of the forward moneyness and we evaluate $v_{l o c}$ such that the "market" model and the simplified model yield the same implied volatility for individual stocks. According to [11, it is enough to take

$$
v_{l o c}(t, x)=\eta^{2}(t, x)+\mathbb{E}\left(\sigma^{2}\left(t, I_{t}\right) \mid S_{t}^{1}=x\right)
$$

where the conditional expectation is approximated using the non-parametric method presented above.

Finally, we fix the correlation coefficient $\rho$ such that the market model and the simplified one have the same ATM implied volatility for the index.

The implied volatilities for the index and for an individual stock obtained by the three models are plotted in Figures 9 and 8 . We also give the difference in basis points between the implied volatilities obtained with the simplified model and the original one in Tables 4 , 5 and 3 . The parameters we use in our numerical experiment are the following :

- $S_{0}^{1}=\cdots=S_{0}^{M}=53$,

- $M, I_{0}$ and the weights $w_{1}, \ldots, w_{M}$ : the same as of the Eurostoxx index at December 21, 2007,

$-r=0.045$,

- Maturity $T=1$ year,

- Number of time steps: $n=10$,

- Number of simulated paths : $N=100000$.

\begin{tabular}{|c|c|c|c|c|c|c|c|c|c|c|c|c|}
\hline Moneyness $\left(\frac{K}{S_{0}}\right)$ & 0.5 & 0.8 & 0.9 & 0.95 & 1 & 1.05 & 1.1 & 1.2 & 1.3 & 1.55 & 1.85 & 2 \\
\hline$\left|\widehat{\sigma}_{\text {simplified }}-\widehat{\sigma}_{\text {original }}\right|$ & 81 & 22 & 16 & 14 & 14 & 17 & 20 & 24 & 24 & 11 & 38 & 17 \\
\hline
\end{tabular}

Table 3: Difference (in bp) the implied volatilities of an individual stock obtained with the simplified model and with the original model.

\begin{tabular}{|c|c|c|c|c|c|c|c|c|c|c|c|c|}
\hline Moneyness $\left(\frac{K}{I_{0}}\right)$ & 0.5 & 0.8 & 0.9 & 0.95 & 1 & 1.05 & 1.1 & 1.2 & 1.3 & 1.55 & 1.85 & 2 \\
\hline$\left|\widehat{\sigma}_{\text {simplified }}-\widehat{\sigma}_{\text {original }}\right|$ & 81 & 22 & 16 & 14 & 14 & 17 & 20 & 24 & 24 & 11 & 38 & 17 \\
\hline
\end{tabular}

Table 4: Difference (in bp) between the implied volatilities of the index obtained with the simplified model and with the original model.

\begin{tabular}{|c|c|c|c|c|c|c|c|c|c|c|c|c|}
\hline Moneyness $\left(\frac{K}{I_{0}}\right)$ & 0.5 & 0.8 & 0.9 & 0.95 & 1 & 1.05 & 1.1 & 1.2 & 1.3 & 1.55 & 1.85 & 2 \\
\hline$\left|\widehat{\sigma}_{\text {reconstruct }}-\widehat{\sigma}_{\text {original }}\right|$ & 10 & 5 & 4 & 3 & 2 & 1 & 2 & 5 & 4 & 1 & 0 & 0 \\
\hline
\end{tabular}

Table 5: Difference (in bp) between the implied volatility of the reconstructed index $\bar{I}^{M}$ in the simplified model and the implied volatility of the index in the original model.

As suggested by Theorems 1 and 2, we see that the original model and the simplified one yield implied volatility curves that are very close to each other, both for the index and for individual stocks. The difference in basis points between the implied volatilities is reasonable, especially between the reconstructed index in the simplified model and the index in the original model. 


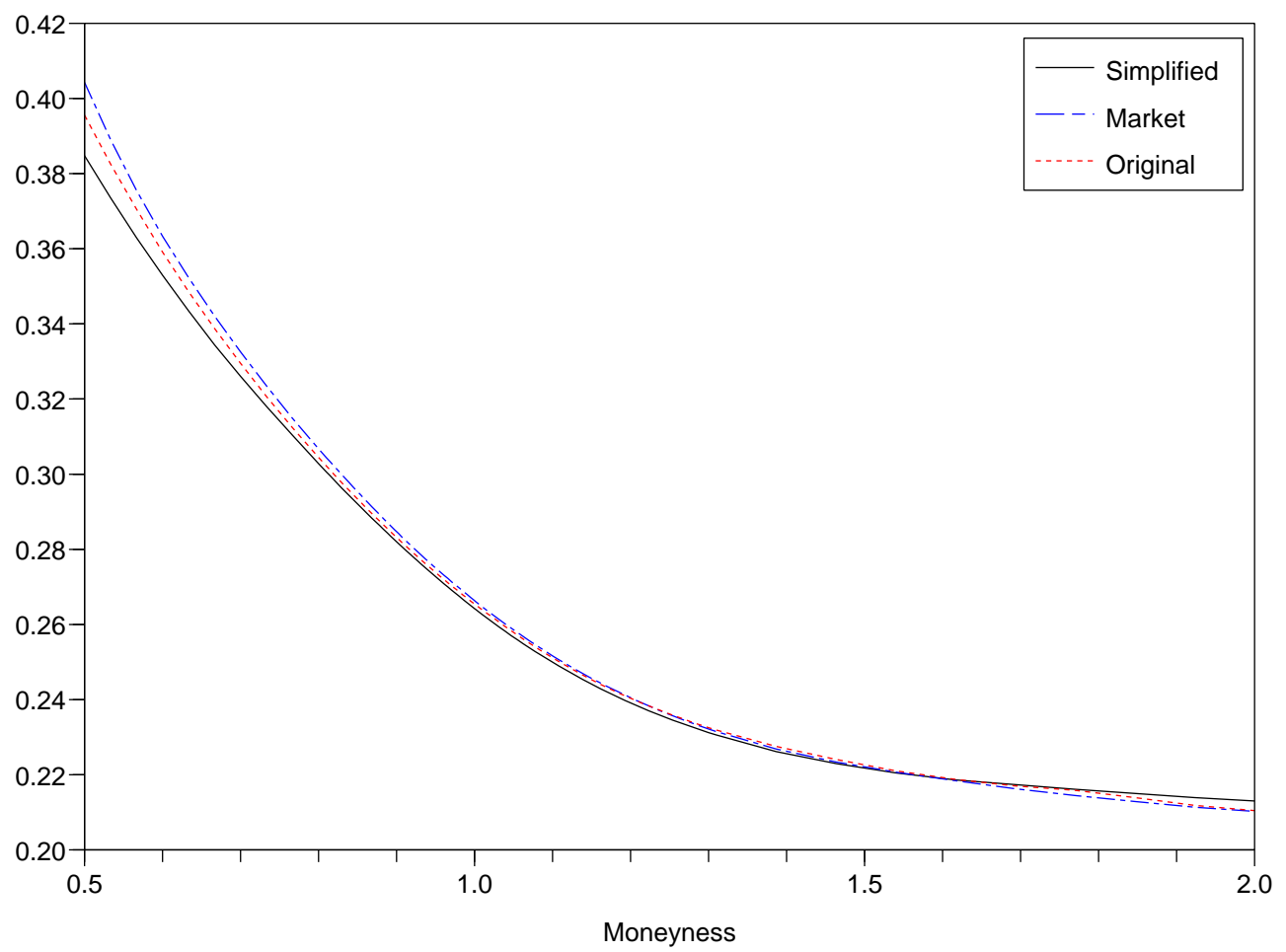

Figure 8: Implied volatility of an individual stock.

Concerning the market model, by construction, we have the same implied volatility for an individual stock as in the simplified model but the implied volatility of the index is far from the simplified one. This phenomenon is well known in practice (see [2, [5, or [7]) : the implied volatility smile of an index is much steeper than the implied volatility smile of an individual stock. The market model of constantly correlated local volatility dynamics for the stocks is unable to retrieve the shape of the index smile. A more sophisticated dependence structure between stocks is needed. Local correlation models provide an extension of the market model in this direction : the correlation at time $t$ between the Brownian motions driving the local volatility dynamics of the stocks is a function $\rho\left(t, I_{t}\right)$ of the index level. But the way this function $\rho$ influences the index smile is not clear at all. Somehow, our models provide another parametrization of the correlation structure in which, the function $\sigma$, that replaces the function $\rho$, can be interpreted as the local volatility of the index. Yet, the individual stocks can still be properly calibrated.

\section{Application: Pricing of a worst-of option}

Apart from handling both the index and its composing stocks, our models are also relevant for the widespread financial products that are sensitive to correlation in the equity world, such as rainbow options.

One example of such products is the worst-of performance option whose payout is referenced to the worst performer in a basket of shares. For a basket of $M$ shares, the payoff of a call with strike $K$ and maturity 


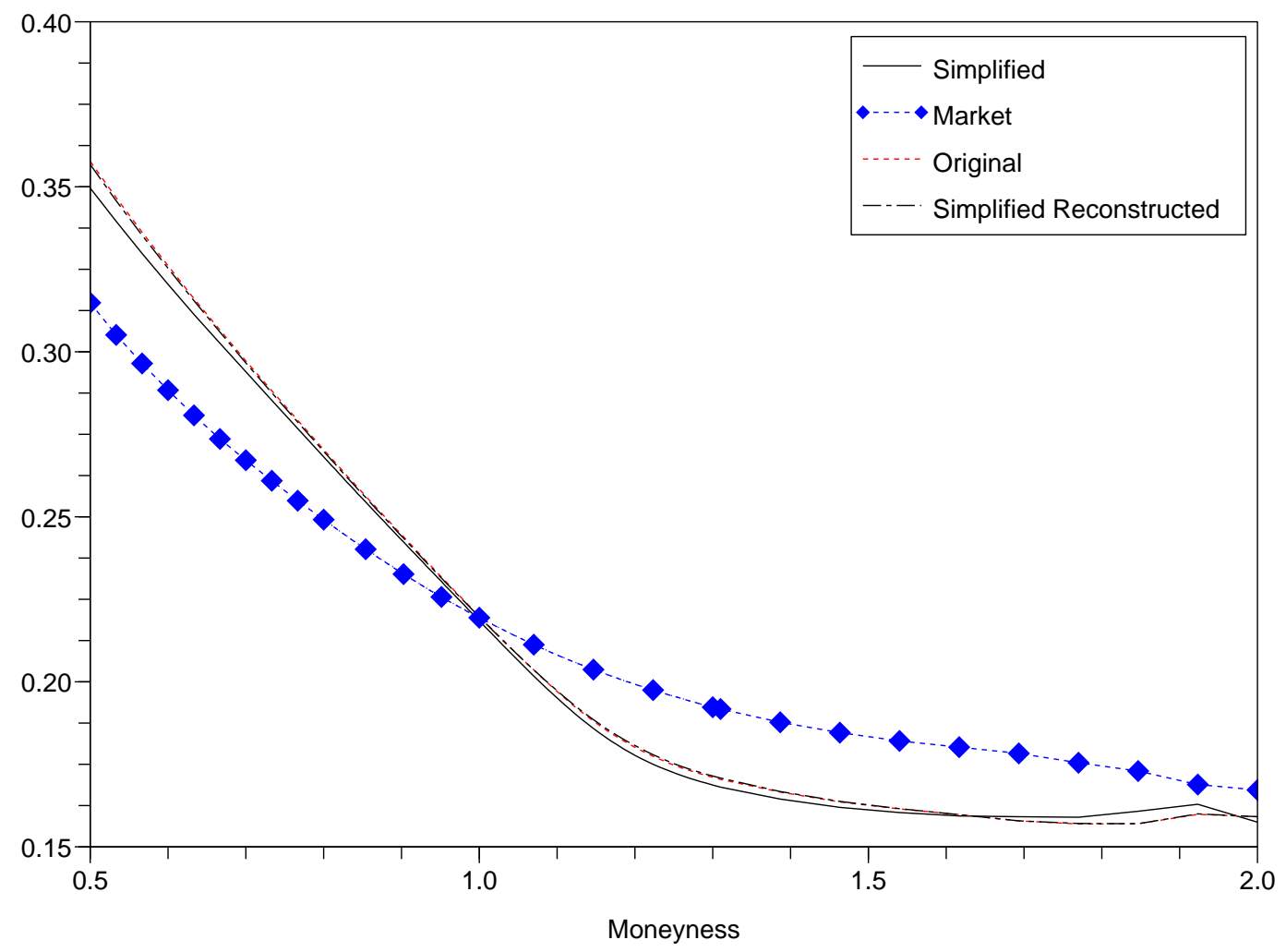

Figure 9: Implied volatility of the index.

$T$ writes $\left(\min _{1 \leq i \leq M} \frac{S_{T}^{i}}{S_{0}^{i}}-K\right)_{+}$. Our objective is to compare the prices obtained by our model to the prices obtained by the market model of constantly correlated stocks. The parameters of the numerical experiment are the same as previously and we set the correlation coefficient $\rho$ such that all the models exhibit the same ATM implied volatility for the index.

The result, as can be seen in Figure 10 is that our prices are always lower than the market model price, especially in the money. Hence, a model with a constant correlation coefficient, calibrated in order to fit the at the money prices of options written on the index, will always overestimate the risks of worst-of options. The reason is that the correlation level needed to fit the at the money prices is very high. Note that the prices obtained with the original model and the simplified one are barely distinguishable from each other. 


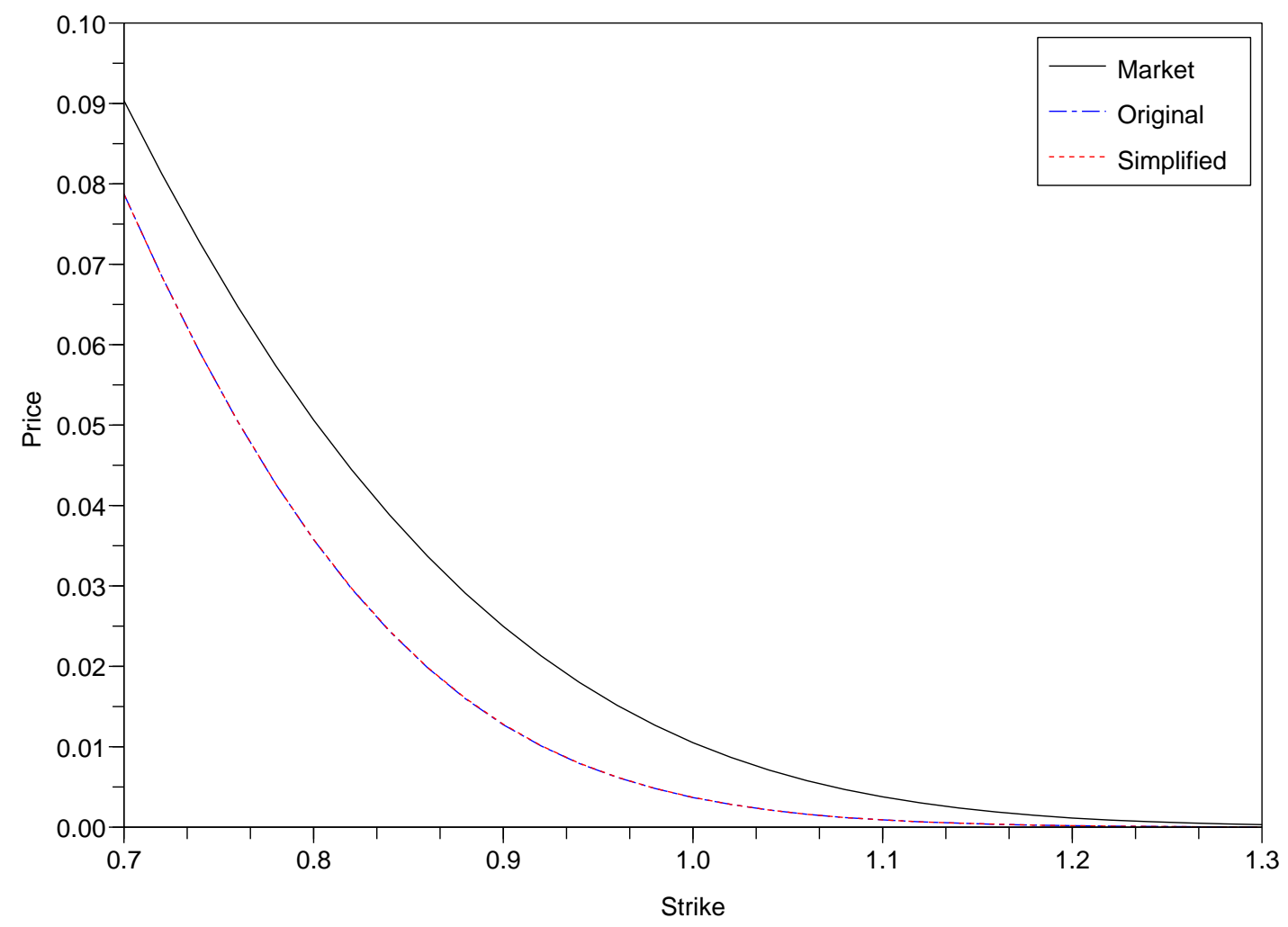

Figure 10: Worst-of price.

\section{Conclusion}

In this paper, we have introduced a new model for describing the joint evolution of an index and its composing stocks. The idea behind our view is that an index is not only a weighted sum of stocks but can also be seen as a market factor that influences their dynamics. In order to have a more tractable model, we have studied the limit when the number of underlying stocks goes to infinity and we have shown that our model reduces to a local volatility model for the index and to a stochastic volatility model with volatility driven by the index for each individual stock. We have discussed calibration issues and proposed a simulation-based technique for the calibration of the stock dynamics, which permits us to fit both index and stocks smiles. The numerical results obtained on real data for the Eurostoxx index are very encouraging, especially for accelerated techniques. We have also compared our models (before and after passing to the limit) to a standard market model consisting of local volatility models for the stocks which are constantly correlated and we have seen that they lead to a steeper index smile. Finally, when considering the pricing of worst-of performance options, which are sensitive to the dependence structure between stocks, we have found that our prices are more aggressive than the prices obtained by the standard market model.

To sum up, we list some properties of our models depending on the options one wishes to handle in the Table below 


\begin{tabular}{|l|l|l|}
\hline \multicolumn{1}{|c|}{ Purpose } & \multicolumn{1}{|c|}{ Simplified model } & \multicolumn{1}{c|}{ Original model } \\
\hline $\begin{array}{l}\text { Options written on } \\
\text {-few }(J<<M) \text { stocks } \\
\text {-the index. }\end{array}$ & $\begin{array}{l}\text {-Simulation of a }(J+1) \text {-dimensional SDE : } \\
\left(I, S^{1}, \ldots, S^{J}\right) .\end{array}$ & $\begin{array}{l}\text {-Simulation of an } M \text {-dimensional SDE : } \\
\left(S^{1, M}, \ldots, S^{M, M}\right) .\end{array}$ \\
& -Exact calibration of $\left(S^{j}\right)_{1 \leq j \leq J}$ and $I$ possible. & $\begin{array}{l}\text {-Exact calibration of }\left(S^{j, M}\right){ }_{1 \leq j \leq J \text { possible }} \\
\text { but requires calibration of all the stocks. } \\
\text {-Approximate calibration of } I^{M} .\end{array}$ \\
\hline $\begin{array}{l}\text { Options written on } \\
\text {-all the stocks } \\
\text {-the index. }\end{array}$ & $\begin{array}{l}\text {-Simulation of an }(M+1) \text {-dimensional SDE : } \\
\left(I, S^{1}, \ldots, S^{M}\right) .\end{array}$ & $\begin{array}{l}\text {-Simulation of an } M \text {-dimensional SDE : } \\
\left(S^{1, M}, \ldots, S^{M, M}\right) . \\
\end{array}$ \\
& -Exact calibration of all the stocks possible. \\
& -Index value $: \bar{I}_{t}^{M}=\sum_{j=1}^{M} w_{j} S_{t}^{j}$. & -Exact calibration of all the stocks possible. \\
& -Approximate calibration of $\bar{I}^{M}$. & \\
\hline
\end{tabular}

Table 6: Which model to use and when.

\section{References}

[1] M. Avellaneda, D. Boyer-Olson, J. Busca, and P. Friz. Reconstructing volatility. Risk, pages 87-91, October 2002.

[2] G. Bakshi, N. Kapadia, and D. Madan. Stock return characteristics, skew laws, and the differential pricing of individual equity options. Review of Financial Studies, 16:101-143, 2003.

[3] H. Berestycki, J. Busca, and I. Florent. An inverse parabolic problem arising in finance. Comptes Rendus de l'Académie des Sciences. Série I. Mathématique, 331(12):965-969, 2000.

[4] H. Berestycki, J. Busca, and I. Florent. Asymptotics and calibration of local volatility models. Quantitative Finance, 2(1):61-69, 2002.

[5] N.P.B. Bollen and R.E. Whaley. Does net buying pressure affect the shape of implied volatility functions? Journal of Finance, 59(2):711-753, 042004.

[6] D. Bosq. Nonparametric statistics for stochastic processes, volume 110 of Lecture Notes in Statistics. Springer-Verlag, New York, second edition, 1998. Estimation and prediction.

[7] N. Branger and C. Schlag. Why is the index smile so steep? Review of Finance, 8(1):109-127, 2004.

[8] P. Cizeau, M. Potters, and J-P. Bouchaud. Correlation structure of extreme stock returns. Quantitative Finance, 1(2):217-222, February 2001.

[9] A. Dermoune. Propagation and conditional propagation of chaos for pressureless gas equations. Probability Theory and Related Fields, 126(4):459-476, 2003.

[10] B. Dupire. Pricing with a smile. Risk, pages 18-20, January 1994.

[11] I. Gyöngy. Mimicking the one-dimensional marginal distributions of processes having an Itô differential. Probability Theory and Related Fields, 71(4):501-516, 1986.

[12] I. Karatzas and S.E. Shreve. Brownian motion and stochastic calculus. Springer-Verlag New-York, second edition, 1991.

[13] P. Lee, L. Wang, and A. Kerim. Index volatility surface via moment-matching techniques. Risk, pages 85-89, December 2003.

[14] F.A. Longstaff and E.S. Schwartz. Valuing American options by simulation: a simple least-squares approach. Review of Financial Studies, 14(1):113-147, 2001. 
[15] S. Méléard. Asymptotic behaviour of some interacting particle systems; McKean-Vlasov and Boltzmann models. In Probabilistic models for nonlinear partial differential equations (Montecatini Terme, 1995), volume 1627 of Lecture Notes in Math., pages 42-95. Springer, Berlin, 1996.

[16] E.A. Nadaraya. On estimating regression. Theory of Probability and its Applications, 9(1):141-142, 1964.

[17] W.F. Sharpe. Capital asset prices: A theory of market equilibrium under conditions of risk. The Journal of Finance, 19(3):425-442, 1964.

[18] A-S. Sznitman. Topics in propagation of chaos. In École d'Été de Probabilités de Saint-Flour XIX - 1989, volume 1464 of Lecture Notes in Math., pages 165-251. Springer, Berlin, 1991.

[19] D. Talay and O. Vaillant. A stochastic particle method with random weights for the computation of statistical solutions of McKean-Vlasov equations. The Annals of Applied Probability, 13(1):140-180, 2003.

[20] G.S. Watson. Smooth regression analysis. Sankhyā (Statistics). The Indian Journal of Statistics. Series $A, 26: 359-372,1964$. 


\section{Appendix}

In order to prove the Theorems 1 and 2, we need the following technical estimation

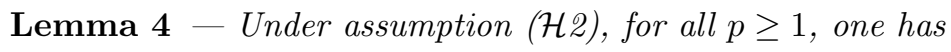

$$
\forall j \in\{1, \ldots, M\}, \quad \sup _{0 \leq t \leq T} \mathbb{E}\left(\left|S_{t}^{j, M}\right|^{2 p}\right) \leq C_{p}
$$

where $C_{p}=\max _{1 \leq j \leq M}\left|S_{0}^{j, M}\right|^{2 p} \exp \left(\left(2 r+(2 p-1)\left(\max _{j \geq 1} \beta_{j}^{2}+1\right) K_{b}^{2}\right) p T\right)$.

Proof : By Itô's lemma one has

$$
\begin{aligned}
\left|S_{t}^{j, M}\right|^{2 p}=\mid & \left.S_{0}^{j, M}\right|^{2 p}+\int_{0}^{t}\left|S_{s}^{j, M}\right|^{2 p}\left((2 p)\left(r-\delta_{j}\right)+p(2 p-1)\left(\beta_{j}^{2} \sigma^{2}\left(s, I_{s}^{M}\right)+\eta_{j}^{2}\left(s, S_{s}^{j, M}\right)\right)\right) d s \\
& +\int_{0}^{t}(2 p)\left|S_{s}^{j, M}\right|^{2 p}\left(\beta_{j} \sigma\left(s, I_{s}^{M}\right) d B_{s}+\eta_{j}\left(s, S_{s}^{j, M}\right) d W_{s}^{j}\right)
\end{aligned}
$$

In order to get rid of the stochastic integral, we use a localization technique : let $\nu_{n}$ be the stopping time defined for each $n \in \mathbb{N}$ by $\nu_{n}:=\inf \left\{t \geq 0 ;\left|S_{t}^{j, M}\right| \geq n\right\}$. Then, using (H(2), one has

$$
\begin{aligned}
\mathbb{E}\left(\left|S_{t \wedge \nu_{n}}^{j, M}\right|^{2 p}\right) & =\left|S_{0}^{j, M}\right|^{2 p}+\mathbb{E}\left(\int_{0}^{t \wedge \nu_{n}}\left|S_{s}^{j, M}\right|^{2 p}\left((2 p)\left(r-\delta_{j}\right)+p(2 p-1)\left(\beta_{j}^{2} \sigma^{2}\left(s, I_{s}^{M}\right)+\eta_{j}^{2}\left(s, S_{s}^{j, M}\right)\right) d s\right)\right. \\
& \leq\left|S_{0}^{j, M}\right|^{2 p}+\left((2 p)\left(r-\delta_{j}\right) \mathbb{1}_{\left\{r-\delta_{j} \geq 0\right\}}+p(2 p-1)\left(\beta_{j}^{2}+1\right) K_{b}^{2}\right) \int_{0}^{t} \mathbb{E}\left(\left|S_{s \wedge \nu_{n}}^{j, M}\right|^{2 p}\right) d s
\end{aligned}
$$

So, by Gronwall's lemma and the fact that the dividends are nonnegative,

$$
\forall t \leq T, \mathbb{E}\left(\left|S_{t \wedge \nu_{n}}^{j, M}\right|^{2 p}\right) \leq\left|S_{0}^{j, M}\right|^{2 p} \exp \left(\left(2 r p+p(2 p-1)\left(\beta_{j}^{2}+1\right) K_{b}^{2}\right) T\right)
$$

Finally, Fatou's lemma permits us to conclude :

$$
\sup _{0 \leq t \leq T} \mathbb{E}\left(\left|S_{t}^{j, M}\right|^{2 p}\right) \leq\left|S_{0}^{j, M}\right|^{2 p} \exp \left(\left(2 r p+p(2 p-1)\left(\beta_{j}^{2}+1\right) K_{b}^{2}\right) T\right) .
$$

Proof of Theorem 1: Using the SDEs (3) and (15), one has

$$
\begin{aligned}
\left|I_{t}^{M}-I_{t}\right|^{2 p}= & \mid r \int_{0}^{t}\left(I_{s}^{M}-I_{s}\right) d s-\int_{0}^{t}\left(\sum_{j=1}^{M} \delta_{j} w_{j} S_{s}^{j, M}-\delta I_{s}\right) d s \\
& +\int_{0}^{t}\left(\sum_{j=1}^{M} \beta_{j} w_{j} S_{s}^{j, M} \sigma\left(s, I_{s}^{M}\right)-\beta I_{s} \sigma\left(s, I_{s}\right)\right) d B_{s}+\left.\sum_{j=1}^{M} w_{j} \int_{0}^{t} S_{s}^{j, M} \eta_{j}\left(s, S_{s}^{j, M}\right) d W_{s}^{j}\right|^{2 p} \\
\leq & 4^{2 p-1}\left(r^{2 p} t^{2 p-1} \int_{0}^{t}\left(I_{s}^{M}-I_{s}\right)^{2 p} d s+t^{2 p-1} \int_{0}^{t}\left(\sum_{j=1}^{M} \delta_{j} w_{j} S_{s}^{j, M}-\delta I_{s}\right)^{2 p} d s\right. \\
& \left.+\left|\int_{0}^{t}\left(\sum_{j=1}^{M} \beta_{j} w_{j} S_{s}^{j, M} \sigma\left(s, I_{s}^{M}\right)-\beta I_{s} \sigma\left(s, I_{s}\right)\right) d B_{s}\right|^{2 p}+\left|\sum_{j=1}^{M} w_{j} \int_{0}^{t} S_{s}^{j, M} \eta_{j}\left(s, S_{s}^{j, M}\right) d W_{s}^{j}\right|^{2 p}\right)
\end{aligned}
$$


Hence, using the Burkholder-Davis-Gundy inequality (see Karatzas and Shreve [12] p. 166), there exists a universal positive constant $K_{p}$ such that

$$
\mathbb{E}\left(\sup _{0 \leq t \leq T}\left|I_{t}^{M}-I_{t}\right|^{2 p}\right) \leq 4^{2 p-1}\left(a_{M}+b_{M}+c_{M}+d_{M}\right)
$$

where

- $a_{M}=r^{2 p} T^{2 p-1} \int_{0}^{T} \mathbb{E}\left(\left(I_{s}^{M}-I_{s}\right)^{2 p}\right) d s$

- $b_{M}=T^{2 p-1} \int_{0}^{T} \mathbb{E}\left(\left(\sum_{j=1}^{M} \delta_{j} w_{j} S_{s}^{j, M}-\delta I_{s}\right)^{2 p}\right) d s$

- $c_{M}=K_{p} T^{p-1} \int_{0}^{T} \mathbb{E}\left(\left(\sum_{j=1}^{M} \beta_{j} w_{j} S_{s}^{j, M} \sigma\left(s, I_{s}^{M}\right)-\beta I_{s} \sigma\left(s, I_{s}\right)\right)^{2 p}\right) d s$

- $d_{M}=K_{p} T^{p-1} \int_{0}^{T} \mathbb{E}\left(\left(\sum_{j=1}^{M}\left(w_{j} S_{s}^{j, M} \eta_{j}\left(s, S_{s}^{j, M}\right)\right)^{2}\right)^{p}\right) d s$

The term $a_{M}$ is the easiest one to handle :

$$
a_{M} \leq r^{2 p} T^{2 p-1} \int_{0}^{T} \mathbb{E}\left(\sup _{0 \leq u \leq s}\left|I_{u}^{M}-I_{u}\right|^{2 p}\right) d s .
$$

Next, using assumption ( $\mathcal{H}(2)$ for the first inequality, Hölder's inequality for the second and lemma 4 for the third, one gets

$$
\begin{aligned}
d_{M} & =K_{p} T^{p-1} \int_{0}^{T} \sum_{j_{1}=1}^{M} \cdots \sum_{j_{p}=1}^{M} \mathbb{E}\left(\prod_{k=1}^{p} w_{j_{k}}^{2}\left(S_{s}^{j_{k}, M}\right)^{2}\left(\eta_{j_{k}}\left(s, S_{s}^{j_{k}, M}\right)\right)^{2}\right) d s \\
& \leq K_{p} K_{b}^{2 p} T^{p-1} \int_{0}^{T} \sum_{j_{1}=1}^{M} \cdots \sum_{j_{p}=1}^{M}\left(\prod_{k=1}^{p} w_{j_{k}}^{2}\right) \mathbb{E}\left(\prod_{k=1}^{p}\left(S_{s}^{j_{k}, M}\right)^{2}\right) d s \\
& \leq K_{p} K_{b}^{2 p} T^{p-1} \int_{0}^{T} \sum_{j_{1}=1}^{M} \cdots \sum_{j_{p}=1}^{M} \prod_{k=1}^{p} w_{j_{k}}^{2}\left(\mathbb{E}\left(\left(S_{s}^{j_{k}, M}\right)^{2 p}\right)\right)^{\frac{1}{p}} d s \\
& \leq K_{p} K_{b}^{2 p} T^{p} C_{p}\left(\sum_{j=1}^{M} w_{j}^{2}\right)^{p}
\end{aligned}
$$

The same arguments enable us to control the term $b_{M}$ : 


$$
\begin{aligned}
b_{M} & =T^{2 p-1} \int_{0}^{T} \mathbb{E}\left(\left(\sum_{j=1}^{M} \delta_{j} w_{j} S_{s}^{j, M}-\delta I_{s}\right)^{2 p}\right) d s \\
& \leq(2 T)^{2 p-1}\left(\int_{0}^{T} \mathbb{E}\left(\left(\sum_{j=1}^{M} \delta_{j} w_{j} S_{s}^{j, M}-\delta I_{s}^{M}\right)^{2 p}\right)+\mathbb{E}\left(\left(\delta I_{s}^{M}-\delta I_{s}\right)^{2 p}\right) d s\right) \\
& \leq(2 T)^{2 p-1} \int_{0}^{T} \mathbb{E}\left(\left(\sum_{j=1}^{M}\left(\delta_{j}-\delta\right) w_{j} S_{s}^{j, M}\right)^{2 p}\right) d s+(2 T)^{2 p-1} \delta^{2 p} \int_{0}^{T} \mathbb{E}\left(\sup _{0 \leq u \leq s}\left|I_{u}^{M}-I_{u}\right|^{2 p}\right) d s \\
& \leq 2^{2 p-1} T^{2 p} C_{p}\left(\sum_{j=1}^{M} w_{j}\left|\delta_{j}-\delta\right|\right)^{2 p}+(2 T)^{2 p-1} \delta^{2 p} \int_{0}^{T} \mathbb{E}\left(\sup _{0 \leq u \leq s}\left|I_{u}^{M}-I_{u}\right|^{2 p}\right) d s .
\end{aligned}
$$

For the remaining term $c_{M}$, we will also need the Lipschitz assumption ( $\mathcal{H}(3)$

$$
\begin{aligned}
c_{M} & =K_{p} T^{p-1} \int_{0}^{T} \mathbb{E}\left(\left(\sum_{j=1}^{M} \beta_{j} w_{j} S_{s}^{j, M} \sigma\left(s, I_{s}^{M}\right)-\beta I_{s} \sigma\left(s, I_{s}\right)\right)^{2 p}\right) d s \\
& \leq 2^{2 p-1} K_{p} T^{p-1}\left(\int_{0}^{T} \mathbb{E}\left(\left(\sum_{j=1}^{M}\left(\beta_{j}-\beta\right) w_{j} S_{s}^{j, M} \sigma\left(s, I_{s}^{M}\right)\right)^{2 p}\right)+\mathbb{E}\left(\left(\beta I_{s}^{M} \sigma\left(s, I_{s}^{M}\right)-\beta I_{s} \sigma\left(s, I_{s}\right)\right)^{2 p}\right) d s\right) \\
& \leq 2^{2 p-1} K_{p} T^{p} K_{b}^{2 p} C_{p}\left(\sum_{j=1}^{M} w_{j}\left|\beta_{j}-\beta\right|\right)^{2 p}+2^{2 p-1} K_{p} T^{p-1}\left(\beta K_{\sigma}\right)^{2 p} \int_{0}^{T} \mathbb{E}\left(\sup _{0 \leq u \leq s}\left|I_{u}^{M}-I_{u}\right|^{2 p}\right) d s .
\end{aligned}
$$

So, combining the inequalities (22), (22), (24) and (25), one obtains

$$
\begin{aligned}
\mathbb{E}\left(\sup _{0 \leq t \leq T}\left|I_{t}^{M}-I_{t}\right|^{2 p}\right) \leq & C_{0}\left(\left(\sum_{j=1}^{M} w_{j}^{2}\right)^{p}+\left(\sum_{j=1}^{M} w_{j}\left|\beta_{j}-\beta\right|\right)^{2 p}+\left(\sum_{j=1}^{M} w_{j}\left|\delta_{j}-\delta\right|\right)^{2 p}\right) \\
& +C_{1} \int_{0}^{T} \mathbb{E}\left(\sup _{0 \leq u \leq s}\left|I_{u}^{M}-I_{u}\right|^{2}\right) d s
\end{aligned}
$$

with $C_{0}=8^{2 p-1} T^{p}\left(T^{p}+K_{p} K_{b}^{2 p}\right) C_{p}$ and $C_{1}=4^{2 p-1}\left(2^{2 p-1} K_{p} T^{p-1}\left(\beta K_{\sigma}\right)^{2 p}+(2 T)^{2 p-1} \delta^{2 p}+r^{2 p} T^{2 p-1}\right)$.

Finally, by means of Gronwall's lemma, we conclude that

$$
\mathbb{E}\left(\sup _{0 \leq t \leq T}\left|I_{t}^{M}-I_{t}\right|^{2 p}\right) \leq C_{T}\left(\left(\sum_{j=1}^{M} w_{j}^{2}\right)^{p}+\left(\sum_{j=1}^{M} w_{j}\left|\beta_{j}-\beta\right|\right)^{2 p}+\left(\sum_{j=1}^{M} w_{j}\left|\delta_{j}-\delta\right|\right)^{2 p}\right)
$$

where

$$
C_{T}=C_{0} e^{C_{1} T}
$$


Proof of Theorem 2: The proof is similar to the previous one :

$$
\begin{aligned}
\left|S_{t}^{j, M}-S_{t}^{j}\right|^{2 p} \leq & 3^{2 p-1}\left(\left(r-\delta_{j}\right)^{2 p} t^{2 p-1} \int_{0}^{t}\left(S_{s}^{j, M}-S_{s}^{j}\right)^{2 p} d s+\left|\int_{0}^{t}\left(S_{s}^{j, M} \eta_{j}\left(s, S_{s}^{j, M}\right)-S_{s}^{j} \eta_{j}\left(s, S_{s}^{j}\right)\right) d W_{s}^{j}\right|^{2 p}\right. \\
& \left.+\beta_{j}^{2 p}\left|\int_{0}^{t}\left(S_{s}^{j, M} \sigma\left(s, I_{s}^{M}\right)-S_{s}^{j} \sigma\left(s, I_{s}\right)\right) d B_{s}\right|^{2 p}\right)
\end{aligned}
$$

hence, using the Burkholder-Davis-Gundy inequality, there exists a constant $K_{p}$ such that

$$
\begin{aligned}
\mathbb{E}\left(\sup _{0 \leq t \leq T}\left|S_{t}^{j, M}-S_{t}^{j}\right|^{2 p}\right) \leq & 3^{2 p-1}\left(\left(r-\delta_{j}\right)^{2 p} T^{2 p-1} \int_{0}^{T} \mathbb{E}\left(\sup _{0 \leq u \leq s}\left|S_{u}^{j, M}-S_{u}^{j}\right|^{2}\right) d s\right. \\
& +K_{p} T^{p-1} \int_{0}^{T} \mathbb{E}\left(\left(S_{s}^{j, M} \eta_{j}\left(s, S_{s}^{j, M}\right)-S_{s}^{j} \eta_{j}\left(s, S_{s}^{j}\right)\right)^{2 p}\right) d s \\
& \left.+K_{p} T^{p-1} \beta_{j}^{2 p} \int_{0}^{T} \mathbb{E}\left(\left(S_{s}^{j, M} \sigma\left(s, I_{s}^{M}\right)-S_{s}^{j} \sigma\left(s, I_{s}\right)\right)^{2 p}\right) d s\right)
\end{aligned}
$$

Using assumption $(\mathcal{H}(4)$, one gets

$$
\int_{0}^{T} \mathbb{E}\left(\left(S_{s}^{j, M} \eta_{j}\left(s, S_{s}^{j, M}\right)-S_{s}^{j} \eta_{j}\left(s, S_{s}^{j}\right)\right)^{2 p}\right) d s \leq K_{\eta}^{2 p} \int_{0}^{T} \mathbb{E}\left(\sup _{0 \leq u \leq s}\left|S_{u}^{j, M}-S_{u}^{j}\right|^{2 p}\right) d s .
$$

Finally, by means of lemma 4 and assumptions $(\mathcal{H}(2)$ and $(\mathcal{H}(3)$,

$$
\begin{aligned}
\int_{0}^{T} \mathbb{E}\left(\left(S_{s}^{j, M} \sigma\left(s, I_{s}^{M}\right)-S_{s}^{j} \sigma\left(s, I_{s}\right)\right)^{2 p}\right) d s \leq & 2^{2 p-1} \int_{0}^{T} \mathbb{E}\left(\left(S_{s}^{j, M}\right)^{2 p}\left(\sigma\left(s, I_{s}^{M}\right)-\sigma\left(s, I_{s}\right)\right)^{2 p}\right) d s . \\
& +2^{2 p-1} \int_{0}^{T} \mathbb{E}\left(\left(\sigma\left(s, I_{s}\right)\right)^{2 p}\left(S_{s}^{j, M}-S_{s}^{j}\right)^{2 p}\right) d s \\
\leq & 2^{2 p-1} C_{2 p}^{\frac{1}{2}} K_{L i p}^{2 p} T \sqrt{\mathbb{E}\left(\sup _{0 \leq t \leq T}\left|I_{t}^{M}-I_{t}\right|^{4 p}\right)} \\
& +2^{2 p-1} K_{b}^{2 p} \int_{0}^{T} \mathbb{E}\left(\sup _{0 \leq t \leq T}\left|S_{s}^{j, M}-S_{s}^{j}\right|^{2 p}\right) d s
\end{aligned}
$$

We deduce using Gronwall's lemma :

$$
\mathbb{E}\left(\sup _{0 \leq t \leq T}\left|S_{t}^{j, M}-S_{t}^{j}\right|^{2 p}\right) \leq \widetilde{C}_{T}^{j} \sqrt{\mathbb{E}\left(\sup _{0 \leq t \leq T}\left|I_{t}^{M}-I_{t}\right|^{4 p}\right)}
$$

where

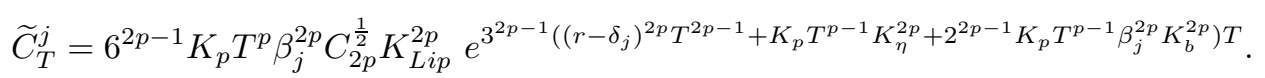

We conclude by Theorem 1 and the sublinearity of the square root function on $\mathbb{R}_{+}$.

We now turn to the $L^{2 p}$-distance between $I^{M}$ and $\bar{I}^{M}$ :

$$
\begin{aligned}
\left|I_{t}^{M}-\bar{I}_{t}^{M}\right|^{2 p} & =\left|\sum_{j=1}^{M} w_{j} S_{t}^{j, M}-\sum_{j=1}^{M} w_{j} S_{t}^{j}\right|^{2 p} \\
& \leq\left(\sum_{j=1}^{M} w_{j}\left|S_{t}^{j, M}-S_{t}^{j}\right|\right)^{2 p} \\
& \leq \sum_{j_{1}=1}^{M} \cdots \sum_{j_{2 p}=1}^{M} \prod_{k=1}^{2 p} w_{j_{k}}\left|S_{t}^{j_{k}, M}-S_{t}^{j_{k}}\right|
\end{aligned}
$$


So, using Hölder inequality, one has

$$
\begin{aligned}
\mathbb{E}\left(\sup _{0 \leq t \leq T}\left|I_{t}^{M}-\bar{I}_{t}^{M}\right|^{2 p} \mid\right) \leq & \sum_{j_{1}=1}^{M} \cdots \sum_{j_{2 p}=1}^{M}\left(\prod_{k=1}^{2 p} w_{j_{k}}\right) \prod_{k=1}^{2 p}\left(\mathbb{E}\left(\sup _{0 \leq t \leq T}\left|S_{t}^{j_{k}, M}-S_{t}^{j_{k}}\right|^{2 p}\right)\right)^{\frac{1}{2 p}} \\
\leq & \left(\sum_{j=1}^{M} w_{j}\right)^{2 p} \max _{1 \leq j \leq M} \widetilde{C}_{T}^{j}\left(\left(\sum_{j=1}^{M} w_{j}^{2}\right)^{p}+\right. \\
& \left.\left(\sum_{j=1}^{M} w_{j}\left|\beta_{j}-\beta\right|\right)^{2 p}+\left(\sum_{j=1}^{M} w_{j}\left|\delta_{j}-\delta\right|\right)^{2 p}\right) .
\end{aligned}
$$

